TRANSACTIONS OF THE

AMERICAN MATHEMATICAL SOCIETY

Volume 361, Number 4, April 2009, Pages 2047-2084

S 0002-9947(08)04694-1

Article electronically published on October 23, 2008

\title{
ENTIRE SOLUTIONS IN BISTABLE REACTION-DIFFUSION EQUATIONS WITH NONLOCAL DELAYED NONLINEARITY
}

\author{
ZHI-CHENG WANG, WAN-TONG LI, AND SHIGUI RUAN
}

\begin{abstract}
This paper is concerned with entire solutions for bistable reactiondiffusion equations with nonlocal delay in one-dimensional spatial domain. Here the entire solutions are defined in the whole space and for all time $t \in \mathbb{R}$. Assuming that the equation has an increasing traveling wave solution with nonzero wave speed and using the comparison argument, we prove the existence of entire solutions which behave as two traveling wave solutions coming from both ends of the $x$-axis and annihilating at a finite time. Furthermore, we show that such an entire solution is unique up to space-time translations and is Liapunov stable. A key idea is to characterize the asymptotic behavior of the solutions as $t \rightarrow-\infty$ in terms of appropriate subsolutions and supersolutions. In order to illustrate our main results, two models of reaction-diffusion equations with nonlocal delay arising from mathematical biology are considered.
\end{abstract}

\section{INTRODUCTION AND MAIN RESULTS}

In this paper, we are concerned with entire solutions of the bistable reactiondiffusion equation with nonlocal delay of the form

$$
\frac{\partial u}{\partial t}=d \Delta u+g\left(u(x, t), \int_{-\tau}^{0} \int_{-\infty}^{\infty} h(y,-s) S(u(x+y, t+s)) d y d s\right)
$$

where $x \in \mathbb{R}, t>0, d>0, \Delta$ is the Laplacian operator on $\mathbb{R}, \tau>0$ is a given constant, and $h \in L^{1}(\mathbb{R} \times[0, \tau])$ is a nonnegative kernel satisfying

(H1) $\int_{0}^{\tau} \int_{0}^{\infty} h(y, s) d y d s=1$ [normalization];

(H2) $h(x, t)=h(-x, t)$ for $(x, t) \in \mathbb{R} \times[0, \tau]$ [spatial symmetry];

(H3) $\int_{0}^{\tau} \int_{0}^{\infty} e^{\lambda y} h(y, s) d y d s<\infty$ for $\lambda \geq 0$ [convergence].

Received by the editors May 10, 2007.

2000 Mathematics Subject Classification. Primary 35K57, 35R10; Secondary 35B40, 34K30, $58 \mathrm{D} 25$.

Key words and phrases. Entire solution, traveling wave solution, reaction-diffusion equation, nonlocal delay, bistable nonlinearity.

The research of the first author was partially supported by NSF of Gansu Province of China (0710RJZA020).

The second author is the corresponding author and was partially supported by NSFC (10571078) and NSF of Gansu Province of China (3ZS061-A25-001).

The research of the third author was partially supported by NSF grants DMS-0412047 and DMS-0715772.

(C)2008 American Mathematical Society Reverts to public domain 28 years from publication 
For the sake of convenience, we set

$$
(h * v)(x, t)=\int_{-\tau}^{0} \int_{-\infty}^{\infty} h(y,-s) v(x+y, t+s) d y d s
$$

for any $v \in C\left(\mathbb{R}^{2}\right)$. Then the spatial symmetry condition (H2) implies that

$$
(h * v)(x, t)=\int_{0}^{\tau} \int_{-\infty}^{\infty} h(y, s) v(x-y, t-s) d y d s .
$$

The nonlinearity is induced by the functions $g$ and $S$, which satisfy the following assumptions:

(F1) $g \in C^{2}([0,1] \times[S(0), S(1)], \mathbb{R})$ and $\partial_{2} g(u, v) \geq 0$ for $(u, v) \in[0,1] \times$ $[S(0), S(1)] ; S \in C^{2}([0,1], \mathbb{R})$ and $S^{\prime}(u) \geq 0$ for $u \in[0,1]$.

(F2) $g(0, S(0))=g(1, S(1))=0, \partial_{1} g(0, S(0))+\partial_{2} g(0, S(0)) S^{\prime}(0)<0$, and $\partial_{1} g(1, S(1))+\partial_{2} g(1, S(1)) S^{\prime}(1)<0$.

We will assume that (1.1) has an increasing traveling wave solution with the wave speed $c$. Hereafter, a traveling wave solution of (1.1) always refers to a pair $(\phi, c)$, where $\phi=\phi(\xi)$ is a function in $\mathbb{R}$ and $c$ is a constant, such that $u(x, t):=\phi(x+c t)$ is a solution of (1.1) and

$$
\lim _{\xi \rightarrow-\infty} \phi(\xi)=0, \quad \lim _{\xi \rightarrow+\infty} \phi(\xi)=1 .
$$

We call $c$ the traveling wave speed and $\phi$ the profile of the wave front. These assumptions about the existence of traveling wave solutions have been justified for a number of important special cases of (1.1) and some more general cases. For example, if $h(x, t)=\delta(t) \delta(x), \delta(\cdot)$ is the Dirac delta function, then (1.1) reduces to the local equation without delay

$$
\frac{\partial u}{\partial t}=d \Delta u+g(u, S(u)), x \in \mathbb{R}, t>0 .
$$

There are many well-known results on traveling wave solutions of (1.3) with bistable nonlinearity; see Fife and McLeod [12, 13, Volpert et al. 39], etc. For the related results on convergency of solutions of (1.3), one can refer to Martin and Smith 27] and Poláčik 31, 32.

If $S(u)=u$ and $h(x, t)=\delta(t-\tau) \delta(x)$, then (1.1) reduces to the local equation with a discrete delay

$$
\frac{\partial u}{\partial t}=d \Delta u+g(u(x, t), u(x, t-\tau)), x \in \mathbb{R}, t>0, \tau>0 .
$$

For Huxley nonlinearity, Schaaf [36] showed that there is exactly one wave speed $c$ such that (1.4) has a nontrivial strictly increasing traveling wave solution. Moreover, he gave the asymptotic behavior of such a traveling wave solution at infinity. Smith and Zhao 38] further proved the global asymptotic stability, Liapunov stability and uniqueness of traveling wave solutions of (1.4) with a bistable nonlinear term.

If $h(x, t)=\delta(t) J(x)$, then (1.1) reduces to the nonlocal equation

$$
\frac{\partial u}{\partial t}=d \Delta u+g\left(u(x, t), \int_{-\infty}^{\infty} J(x-y) S(u(y, t)) d y\right), x \in \mathbb{R}, t>0 .
$$

Chen [7] proved the existence, uniqueness and global asymptotic stability of traveling wave solutions of (1.5) by developing the so-called squeezing technique. 
If $g(u, v)=-\alpha u+v, S(u)=b(u)$ and $h(x, t)=\delta(t-\tau) J(x)$, then (1.1) reduces to the nonlocal equation

$$
\frac{\partial u}{\partial t}=d \Delta u-\alpha u(x, t)+\int_{-\infty}^{\infty} J(x-y) b(u(y, t-\tau)) d y, x \in \mathbb{R}, t>0, \tau>0
$$

which was studied by $\mathrm{Ma}$ and $\mathrm{Wu}[26$. Under the bistable assumption, they proved the existence, uniqueness and global asymptotic stability of traveling wave solutions of (1.6).

Recently, Wang et al. [42] studied the reaction advection diffusion equation with nonlocal delay and a bistable nonlinear term of the form

$$
\frac{\partial u}{\partial t}=d \Delta u+B \frac{\partial u}{\partial x}+g\left(u(x, t),\left(\int_{-\tau}^{0} \int_{-\infty}^{\infty} h(x-y,-s) S(u(y, t+s)) d y d s\right)(x, t)\right),
$$

and established the existence, uniqueness and global asymptotic stability of traveling wave solutions of (1.7).

Since time delay and nonlocality play very important roles in biological and epidemiological models (see Britton [5] and Ruan [34]), they have a crucial effect on the dynamics of the equation (1.1); see Gourley et al. [19, Li et al. [23, 24, Wang and $\mathrm{Li}$ [40] and $\mathrm{Wu}$ [45]. There has been significant progress in the study of traveling wave solutions for both bistable and monostable equations; see, for example, Ai [1], Ashwin et al. [2, Billingham [4], Faria et al. [14, 15], Gourley and Kuang 17, 18, Liang and $\mathrm{Wu}$ 25, Ou and $\mathrm{Wu}$ 29, Ruan and Xiao 35, Wang et al. [41, 43, Wu and Zou [46, Zou [48, and the references cites therein.

On the other hand, it has been observed that traveling wave solutions are special examples of the so-called entire solutions that are defined in the whole space and for all time $t \in \mathbb{R}$. In particular, Chen and Guo 8 , Fukao et al. 16, Guo and Morita [20, Hamel and Nadirashvili [21, 22, Morita and Ninomiya 28. and Yagisita 47] have shown that the study of entire solutions is essential for a full understanding of the transient dynamics and the structure of the global attractors. These studies showed the great diversity of different types of entire solutions of reaction-diffusion equations in the absence of time delay. By constructing a global invariant manifold with asymptotic stability, Yagisita [4]. proved that, for the bistable equation, there exists an entire solution which behaves as two traveling wave solutions coming from both sides of the $x$-axis and annihilating in a finite time. The stability and uniqueness of entire solutions were also considered. Yagisita's argument was substantially simplified by Fukao et al. [16, and the existence of an entire solution emanating from the unstable standing pulse solution of (1.1) was also obtained. For the Fisher-KPP equation, Hamel and Nadirashvili 21] established five-dimensional, four-dimensional and three-dimensional manifolds of entire solutions, respectively, by combining two traveling wave solutions with different speeds and coming from both sides of the real axis and some spatially independent solution. In 22], Hamel and Nadirashvili further considered the existence of entire solutions of the FisherKPP equation in high-dimensional spaces and obtained an amazingly rich class of entire solutions. Chen and Guo [8] and Guo and Morita [20] developed a unified approach based on the comparison principle to find entire solutions for both the bistable and monostable cases. Furthermore, Chen et al. [9] considered entire solutions of reaction-diffusion equations with bistable nonlinearities for the case 
$c=0$. Morita and Ninomiya 28 showed some novel entire solutions which are completely different from these observed in $8,16,20,21,22,47$.

However, the above mentioned results are only concerned with entire solutions of reaction-diffusion equations in the absence of time delay and nonlocality. The issue of the existence of entire solutions for a general bistable equation with nonlocal delay including the Huxley equation [36 and the single population with stage structure and distributed maturation delay [3] is still open. The goal of this paper is to resolve this issue.

In this paper, we consider some new types of entire solutions of (1.1). Our method is to construct appropriate supersolutions and subsolutions and then show the existence of the desired entire solutions by comparison and the continuity of the semiflow, which is inspired by Chen and Guo [8] and Guo and Morita 20] and are done in Section 4. Before doing that, we study the asymptotic behavior of traveling wave solutions at infinity in Section 3. Furthermore, the uniqueness and stability of such an entire solution are established in Section 5.

Throughout the paper, we always assume that (H1), (H2), (H3), (F1) and (F2) hold. Now we state our main results in this paper.

Theorem 1.1. Assume that equation (1.1) admits an increasing traveling wave solution $\phi$ with speed $c>0$. Then for any given constants $\theta_{1}$ and $\theta_{2}$ there exists a solution $\Phi(x, t)$ of (1.1) defined for all $(x, t) \in \mathbb{R}^{2}$ such that $0<\Phi(x, t)<1$, $\frac{\partial \Phi}{\partial t}>0$ and

$$
\lim _{t \rightarrow-\infty}\left\{\sup _{x \geq 0}\left|\Phi(x, t)-\phi\left(x+c t+\theta_{1}\right)\right|+\sup _{x \leq 0}\left|\Phi(x, t)-\phi\left(-x+c t+\theta_{2}\right)\right|\right\}=0 .
$$

In particular, the entire solution is Liapunov stable. Furthermore, assume that $h(x, t)=J(x) \delta(t-\tau)$ and $\widetilde{\Phi}(x, t)$ is another solution of (1.1) satisfying $0<$ $\widetilde{\Phi}(x, t)<1$ and

$\left(\mathbf{U}^{+}\right)$there exist constants $a>0$ and $T_{0} \in \mathbb{R}$, and functions $l(\cdot)$ and $r(\cdot)$ such that for all $t \leq T_{0}$ and $s \in[-\tau, 0]$,

$$
\begin{cases}\widetilde{\Phi}(x, t+s) \geq \beta_{0} & \forall x \in(-\infty, l(t)] \cup[r(t), \infty), \\ \widetilde{\Phi}(x, t+s) \leq \alpha_{0} & \forall x \in[\min \{l(t)+a, r(t)-a\}, \max \{l(t)+a, r(t)-a\}],\end{cases}
$$

where $\alpha_{0}$ and $\beta_{0}$ are constants satisfying

$$
g(u, S(u))<0 \text { in } u \in\left(0, \alpha_{0}\right] \text { and } g(u, S(u))>0 \text { in } u \in\left[\beta_{0}, 1\right) .
$$

Then $\widetilde{\Phi}(x, t)$ is also Liapunov stable, and there exist $x_{0} \in \mathbb{R}$ and $t_{0} \in \mathbb{R}$ such that

$$
\widetilde{\Phi}(x, t)=\Phi\left(x+x_{0}, t+t_{0}\right) \quad \text { for any }(x, t) \in \mathbb{R}^{2} .
$$

Theorem 1.2. Assume that equation (1.1) admits an increasing traveling wave solution $\phi$ with wave speed $c<0$. Then for any given constants $\theta_{1}$ and $\theta_{2}$ there exists a solution $\Phi(x, t)$ of (1.1) defined for all $(x, t) \in \mathbb{R}^{2}$ such that $0<\Phi(x, t)<1$, $\frac{\partial \Phi}{\partial t}<0$ and

$$
\lim _{t \rightarrow-\infty}\left\{\sup _{x \geq 0}\left|\Phi(x, t)-\phi\left(-x+c t+\theta_{1}\right)\right|+\sup _{x \leq 0}\left|\Phi(x, t)-\phi\left(x+c t+\theta_{2}\right)\right|\right\}=0 .
$$


In particular, the entire solution is Liapunov stable. Furthermore, assume that $h(x, t)=J(x) \delta(t-\tau)$ and $\widetilde{\Phi}(x, t)$ is another solution of (1.1) satisfying $0<$ $\widetilde{\Phi}(x, t)<1$ and

$\left(\mathbf{U}^{-}\right)$there exist constants $a>0$ and $T_{0} \in \mathbb{R}$, and functions $l(\cdot)$ and $r(\cdot)$ such that for all $t \leq T_{0}$ and $s \in[-\tau, 0]$,

$$
\begin{cases}\widetilde{\Phi}(x, t+s) \geq \beta_{0} & \forall x \in[\min \{l(t)+a, r(t)-a\}, \max \{l(t)+a, r(t)-a\}], \\ \widetilde{\Phi}(x, t+s) \leq \alpha_{0} & \forall x \in(-\infty, l(t)] \cup[r(t), \infty),\end{cases}
$$

where $\alpha_{0}$ and $\beta_{0}$ are constants satisfying

$$
g(u, S(u))<0 \text { in } u \in\left(0, \alpha_{0}\right] \text { and } g(u, S(u))>0 \text { in } u \in\left[\beta_{0}, 1\right) .
$$

Then $\widetilde{\Phi}(x, t)$ is also Liapunov stable, and there exist $x_{0} \in \mathbb{R}$ and $t_{0} \in \mathbb{R}$ such that

$$
\widetilde{\Phi}(x, t)=\Phi\left(x+x_{0}, t+t_{0}\right) \quad \text { for any }(x, t) \in \mathbb{R}^{2} .
$$

Remark 1.3. If there exists $\tau_{0} \in(0, \tau)$ such that $h(x, t)$ satisfies $\int_{\tau_{0}}^{\tau} \int_{-\infty}^{\infty} h(x, t) d x d t$ $=1$, that is, $\int_{0}^{\tau_{0}} \int_{-\infty}^{\infty} h(x, t) d x d t=0$, then $\widetilde{\Phi}(x, t)$ in Theorems 1.1 and 1.2 is still a translation of $\Phi$, respectively. See Remark 5.9.

Remark 1.4. For the case $h(x, t)=\delta(x) \delta(t)$, Theorem 1.1 concludes Theorem 1.1(i) of Fukao et al. [16, Theorem 1.1 of Guo and Morita 20] and Theorem 1.1 of Yagisita [47. Theorem 1.2 concludes Theorems 1 and 2 of Chen and Guo [8].

In the following, we give two applications of Theorems 1.1 and 1.2 ,

Example 1.5. Consider the typical Huxley nonlinearity

$$
g(u, v)= \begin{cases}u(1-u)(v-a) & \text { for } 0 \leq u \leq 1, v \in \mathbb{R} \\ u(1-u)(u-a) & \text { otherwise }\end{cases}
$$

with $a \in(0,1), a \neq \frac{1}{2}$. This is a special case of equation (1.1) with $S(u)=u$ and $h(x, t)=\delta(x) \delta(t-\tau), \tau \geq 0$. Following Theorem 3.13 of Schaaf [36, p. 603], we know that (1.1) has an increasing traveling wave solution with speed $c>0$ if $a \in\left(0, \frac{1}{2}\right)$ and an increasing traveling wave solution with speed $c<0$ if $a \in\left(\frac{1}{2}, 1\right)$. Thus, Theorems 1.1 and 1.2 hold for (1.1) with the Huxley nonlinearity when $a \in\left(0, \frac{1}{2}\right)$ and $a \in\left(\frac{1}{2}, 1\right)$, respectively.

Example 1.6. Al-Omari and Gourley [3] derived a nonlocal reaction-diffusion model for a single population with stage structure and distributed maturation delay, namely,

$$
\left\{\begin{aligned}
\frac{\partial u_{i}}{\partial t}= & D_{i} \Delta u_{i}+b\left(u_{m}(x, t)\right)-\gamma u_{i}(x, t) \\
& -\int_{0}^{\tau} \int_{\Omega} G(x, y, s) f(s) e^{-\gamma s} b\left(u_{m}(y, t-s)\right) d y d s \\
\frac{\partial u_{m}}{\partial t}= & D_{m} \Delta u_{m}-d\left(u_{m}(x, t)\right) \\
& +\int_{0}^{\tau} \int_{\Omega} G(x, y, s) f(s) e^{-\gamma s} b\left(u_{m}(y, t-s)\right) d y d s
\end{aligned}\right.
$$


where $\int_{0}^{\tau} f(s) d s=1, \Omega \subset \mathbb{R}^{N}$ is open and bounded, $G(x, y, t)$ is the solution subject to the homogeneous Neumann boundary condition of

$$
\frac{\partial G}{\partial t}=D_{i} \Delta_{x} G, \quad G(x, y, 0)=\delta(x-y) .
$$

If the bounded domain $\Omega$ is replaced by the whole real line $(-\infty, \infty)$, then the second equation of (1.10) reduces to

$$
\begin{aligned}
\frac{\partial u_{m}}{\partial t}= & D_{m} \Delta u_{m}-d\left(u_{m}(x, t)\right) \\
& +\int_{0}^{\tau} \int_{-\infty}^{\infty} \frac{1}{\sqrt{4 \pi D_{i} s}} e^{\frac{-(x-y)^{2}}{4 D_{i} s}} f(s) e^{-\gamma s} b\left(u_{m}(y, t-s)\right) d y d s .
\end{aligned}
$$

$\mathrm{Ma}$ and $\mathrm{Wu}[26$ ] considered a special case (1.6) of (1.11). In [42], we showed that (1.11) has an increasing traveling wave solution under the following conditions:

(C1) There exist $0 \leq a_{1}<a_{2}<a_{3}$ such that $\varepsilon b\left(a_{i}\right)-d\left(a_{i}\right)=0, i=1,2,3$; $\varepsilon b(u)-d(u)<0$ for $u \in\left(a_{1}, a_{2}\right) ; \varepsilon b(u)-d(u)>0$ for $u \in\left(a_{2}, a_{3}\right)$, where $\varepsilon=\int_{0}^{\tau} f(s) e^{-\gamma s} d s$.

(C2) $b(\cdot), d(\cdot) \in C^{2}\left(\left[a_{1}, a_{3}\right]\right), b^{\prime}(\cdot) \geq 0, \varepsilon b^{\prime}\left(a_{1}\right)<d^{\prime}\left(a_{1}\right), \varepsilon b^{\prime}\left(a_{2}\right)>d^{\prime}\left(a_{2}\right)$, $\varepsilon b^{\prime}\left(a_{3}\right)<d^{\prime}\left(a_{3}\right)$.

Assume that $(\mathrm{C} 1)$ and $(\mathrm{C} 2)$ hold. If the wave speed of the traveling wave solution of (1.11) is nonzero, then the existence and stability of the entire solutions of (1.11) follow from Theorems 1.1 and 1.2. If there exists $\tau_{0} \in(0, \tau)$ such that $\int_{\tau_{0}}^{\tau} f(s) d s=$ 1 , which contains the case $f(s)=\delta(t-\tau)$, then the uniqueness of the entire solutions in Theorems 1.1 and 1.2 and Remark 1.3 are valid for (1.11).

\section{Preliminaries}

In this section, we state some definitions and establish the comparison theorem for (1.1), which is needed in the sequel.

Let $X=B U C(\mathbb{R}, \mathbb{R})$ be the Banach space of all bounded and uniformly continuous functions from $\mathbb{R}$ into $\mathbb{R}$ with the usual supremum norm. Let $X^{+}=$ $\{\varphi \in X: \varphi(x) \geq 0, x \in \mathbb{R}\}$. It is easy to see that $X^{+}$is a closed cone of $X$ and $X$ is a Banach lattice under the partial ordering induced by $X^{+}$. By 10 , Theorem 1.5 , it then follows that the $X$-realization $d \Delta_{X}$ of $d \Delta$ generates a strongly continuous analytic semigroup $T(t)$ on $X$ and $T(t) X^{+} \subset X^{+}, t \geq 0$. Moreover, we have

$$
\begin{aligned}
T(t) \varphi(x) & =\frac{1}{\sqrt{4 \pi d t}} \int_{-\infty}^{\infty} \exp \left(-\frac{(x-y)^{2}}{4 d t}\right) \varphi(y) d y, \\
x & \in \mathbb{R}, t>0, \varphi(\cdot) \in X .
\end{aligned}
$$

Let $C=C([-\tau, 0], X)$ be the Banach space of continuous functions from $[-\tau, 0]$ into $X$ with the supremum norm and let $C^{+}=\left\{\varphi \in C: \varphi(s) \in X^{+}, s \in[-\tau, 0]\right\}$. Then $C^{+}$is a positive cone of $C$. As usual, we identify an element $\varphi \in C$ as a function from $\mathbb{R} \times[-\tau, 0]$ into $\mathbb{R}$ defined by $\varphi(x, s)=\varphi(s)(x)$. For any continuous function $w:[-\tau, b) \rightarrow X, b>0$, we define $w_{t} \in C, t \in[0, b)$, by $w_{t}(s)=w(t+s), s \in[-\tau, 0]$. Then $t \mapsto w_{t}$ is a continuous function from $[0, b)$ to $C$. For any $\varphi \in C_{[0,1]}=\{\varphi \in C: \varphi(x, s) \in[0,1], x \in \mathbb{R}, s \in[-\tau, 0]\}$, define

$$
F(\varphi)(x)=g\left(\varphi(x, 0), \int_{-\tau}^{0} \int_{-\infty}^{\infty} h(x-y,-s) S(\varphi(y, s)) d y d s\right) .
$$


By the global Lipschitz continuity of $g(\cdot, \cdot)$ on $[0,1] \times[S(0), S(1)]$ and $S(\cdot)$ on $[0,1]$, we can verify that $F(\varphi) \in X$ and $F: C_{[0,1]} \rightarrow X$ is globally Lipschitz continuous.

Definition 2.1. A continuous function $v:[-\tau, b) \rightarrow X, b>0$, is called a supersolution (subsolution) of (1.1) on $[0, b)$ if and only if

$$
v(t) \geq(\leq) T(t-s) v(s)+\int_{s}^{t} T(t-r) F\left(v_{r}\right) d r
$$

for all $0 \leq s<t<b$. If $v$ is both a supersolution and a subsolution on $[0, b)$, then it is said to be a mild solution of (1.1).

Definition 2.2. A function $v:(-\infty, T) \rightarrow X, T \in \mathbb{R}$, is called a supersolution (subsolution) of (1.1) on $(-\infty, T)$ if and only if for any $T^{\prime}<T, w(t):\left[-\tau, T-T^{\prime}\right) \rightarrow$ $X$ defined by $w(t)=v\left(t+T^{\prime}\right)$ for $t \in\left[-\tau, T-T^{\prime}\right)$ is a supersolution (subsolution) of (1.1) on $\left[0, T-T^{\prime}\right)$.

In [42, 43], we have established the following existence and comparison result.

Theorem 2.3. For any $\varphi \in C_{[0,1]}$, (1.1) has a unique mild solution $u(x, t ; \varphi)$ on $[0, \infty)$ which is a classical solution to (1.1) for $(x, t) \in \mathbb{R} \times(\tau, \infty)$. Furthermore, for any pair of supersolutions $\varphi^{+}(x, t)$ and subsolutions $\varphi^{-}(x, t)$ of (1.1) on $[0, b)$ with $0 \leq \varphi^{+}(x, t), \varphi^{-}(x, t) \leq 1$ for $x \in \mathbb{R}, t \in[-\tau, b)$, and $\varphi^{+}(x, s) \geq \varphi^{-}(x, s)$ for $x \in \mathbb{R}, s \in[-\tau, 0], 0<b \leq \infty$, we have $\varphi^{+}(x, t) \geq \varphi^{-}(x, t)$ for $x \in \mathbb{R}, 0 \leq t<b$ and

$$
\varphi^{+}(x, t)-\varphi^{-}(x, t) \geq \Theta\left(J, t-t_{0}\right) \int_{z}^{z+1}\left(\varphi^{+}\left(y, t_{0}\right)-\varphi^{-}\left(y, t_{0}\right)\right) d y
$$

for any $J \geq 0, x$ and $z \in \mathbb{R}$ with $|x-z| \leq J$, and $t>t_{0} \geq 0$, where

$$
\Theta(J, t)=\frac{1}{\sqrt{4 \pi d t}} \exp \left(-L_{1} t-\frac{(J+1)^{2}}{4 d t}\right), J \geq 0, t>0,
$$

and $L_{1}=\max _{(u, v) \in[0,1] \times[S(0), S(1)]}\left|\partial_{1} g(u, v)\right|$. In particular, if there exists $x_{0} \in \mathbb{R}$ such that $\varphi^{+}\left(x_{0}, 0\right)>\varphi^{-}\left(x_{0}, 0\right)$, then $\varphi^{+}(x, t)>\varphi^{-}(x, t)$ for any $x \in \mathbb{R}$ and $t>0$.

Remark 2.4. For $\tau=0$, that is, for the equation without delay, Theorem 2.3 still holds.

\section{AsYmptotic BehaVior of tRAVELING WAVE SOLUtions}

In this section, we will discuss the asymptotic behavior of traveling wave solutions of (1.1) at infinity. Define a function

$$
\begin{aligned}
G(\lambda) & =\int_{0}^{\tau} \int_{-\infty}^{\infty} h(y, s) e^{-\lambda(y+c s)} d y d s \\
& =\int_{0}^{\tau} \int_{0}^{\infty} h(y, s)\left(e^{\lambda y}+e^{-\lambda y}\right) e^{-\lambda c s} d y d s, \lambda \in \mathbb{C},
\end{aligned}
$$

where $c \in \mathbb{R}$ is a constant. Since $e^{-i \operatorname{Im} \lambda y}$ and $e^{-i \operatorname{Im} \lambda c s}$ are bounded, $G(\lambda)$ is well defined in $\mathbb{C}$. Obviously, $G(0)=1$.

Lemma 3.1. For $\lambda \in \mathbb{R}, G(\lambda)$ satisfies

$$
\frac{\partial}{\partial \lambda} G(\lambda)=\int_{0}^{\tau} \int_{0}^{\infty} h(y, s)\left[(y-c s) e^{\lambda(y-c s)}-(y+c s) e^{-\lambda(y+c s)}\right] d y d s
$$


and

$$
\frac{\partial^{2}}{\partial \lambda^{2}} G(\lambda)=\int_{0}^{\tau} \int_{0}^{\infty} h(y, s)\left[(y-c s)^{2} e^{\lambda(y-c s)}+(y+c s)^{2} e^{-\lambda(y+c s)}\right] d y d s>0 .
$$

The lemma can be proved by condition (H3) and Lebesgue's dominated convergence theorem, so its proof is omitted.

Define two complex functions $\Delta_{0}(\lambda)$ and $\Delta_{1}(\lambda)$ by

$$
\begin{aligned}
& \Delta_{0}(\lambda)=d \lambda^{2}-c \lambda+\partial_{1} g(0, S(0))+\partial_{2} g(0, S(0)) S^{\prime}(0) G(\lambda), \\
& \Delta_{1}(\lambda)=d \lambda^{2}-c \lambda+\partial_{1} g(1, S(1))+\partial_{2} g(1, S(1)) S^{\prime}(1) G(\lambda),
\end{aligned}
$$

where $\lambda \in \mathbb{C}$. Then it is easy to see that the following result holds.

Lemma 3.2. The equation $\Delta_{i}(\lambda)=0$ has two real roots $\lambda_{i 1}<0$ and $\lambda_{i 2}>0$ such that

$$
\Delta_{i}(\lambda)= \begin{cases}>0 & \text { for } \lambda<\lambda_{i 1} \\ <0 & \text { for } \lambda \in\left(\lambda_{i 1}, \lambda_{i 2}\right) \\ >0 & \text { for } \lambda>\lambda_{i 2}\end{cases}
$$

where $i=0,1$.

In the following, we investigate the asymptotic behavior of traveling wave solutions at infinity. Our method is similar to that of Carr and Chmaj [6] which has been used by Wang et al. 43] (see also Diekmann and Kaper [11]). We first provide a technical lemma about the asymptotic behavior of a positive decreasing function, which is given by Carr and Chmaj [6, Proposition 2.3] and is important to prove our results.

Lemma 3.3. Let $\ell(\lambda)=\int_{0}^{\infty} u(\xi) e^{-\lambda \xi} d \xi$ with $u(\xi)$ being a positive decreasing function. Assume that $\ell$ has the representation

$$
\ell(\lambda)=\frac{E(\lambda)}{(\lambda+\alpha)^{k+1}}
$$

where $k>-1$ and $E$ is analytic in the strip $-\alpha \leq R e \lambda<0$. Then

$$
\lim _{\xi \rightarrow+\infty} \frac{u(\xi)}{\xi^{k} e^{-\alpha \xi}}=\frac{E(-\alpha)}{\Gamma(\alpha+1)} .
$$

Lemma 3.4. Assume further that $\tilde{\phi}(t)$ is an increasing traveling wave solution of (1.1) satisfying $0<\tilde{\phi}(t)<1$ and (1.2). Then $\tilde{\phi}^{\prime}(t)>0$ and $\lim _{t \rightarrow \pm \infty} \tilde{\phi}^{\prime}(t)=0$.

The proof of Lemma 3.4 follows from Theorem 2.3 and a similar argument to Lemma 2.5 of Smith and Zhao [38].

Theorem 3.5. Assume that $\phi(t)$ is an increasing traveling wave solution of (1.1) satisfying (1.2) with wave speed $c \in \mathbb{R}$. Then

(i) $\lim _{t \rightarrow-\infty} e^{-\lambda_{02} t} \phi(t)=a_{02}, \lim _{t \rightarrow-\infty} e^{-\lambda_{02} t}(h * \phi)(t)=a_{02} G\left(\lambda_{02}\right)$ and $\lim _{t \rightarrow-\infty} e^{-\lambda_{02} t} \phi^{\prime}(t)=\lambda_{02} a_{02}$, where

$$
(h * \phi)(t)=\int_{0}^{\tau} \int_{-\infty}^{+\infty} h(y, s) \phi(t-y-c s) d y d s
$$

and $a_{02}>0$ is a constant.

(ii) $\lim _{t \rightarrow \infty} e^{-\lambda_{11} t}(1-\phi(t))=a_{11}, \lim _{t \rightarrow \infty} e^{-\lambda_{11} t}((1-(h * \phi)(t)))=$ $a_{11} G\left(\lambda_{11}\right)$ and $\lim _{t \rightarrow \infty} e^{-\lambda_{11} t} \phi^{\prime}(t)=-\lambda_{11} a_{11}$, where $a_{11}>0$ is a constant. 
Proof. Let $U(t)=1-\phi(t)$ and define $V(t)=\int_{0}^{\tau} \int_{-\infty}^{\infty} h(z, r) U(t-c r-z) d z d r$. Since $U(t)$ satisfies $-d U^{\prime \prime}(t)+c U^{\prime}(t)=-g(1-U(t),(h * S(1-U))(t))$, then

$$
U(t)=\frac{1}{d\left(\lambda_{2}-\lambda_{1}\right)}\left[\int_{-\infty}^{t} e^{\lambda_{1}(t-s)} H(U)(s) d s+\int_{t}^{\infty} e^{\lambda_{2}(t-s)} H(U)(s) d s\right],
$$

where $H(U)(t)=-g(1-U(t),(h * S(1-U))(t))+L_{1} U(t), L_{1}$ is defined in Theorem 2.3. and

$$
\lambda_{1}=\frac{c-\sqrt{c^{2}+4 d L_{1}}}{2 d}, \quad \lambda_{2}=\frac{c+\sqrt{c^{2}+4 d L_{1}}}{2 d} .
$$

By virtue of $-g(1-U(t),(h * S(1-U))(t))+L_{1} U(t) \geq 0$, for $\beta=\max \left\{-\lambda_{1}, \lambda_{2}\right\}$,

$$
\begin{aligned}
\frac{d}{d t} & {\left[U(t) e^{\beta t}\right] } \\
= & \frac{e^{\beta t}}{d\left(\lambda_{2}-\lambda_{1}\right)}\left[\beta \int_{-\infty}^{t} e^{\lambda_{1}(t-s)} H(U)(s) d s+\beta \int_{t}^{\infty} e^{\lambda_{2}(t-s)} H(U)(s) d s\right] \\
& +\frac{e^{\beta t}}{d\left(\lambda_{2}-\lambda_{1}\right)}\left[\lambda_{1} \int_{-\infty}^{t} e^{\lambda_{1}(t-s)} H(U)(s) d s+\lambda_{2} \int_{t}^{\infty} e^{\lambda_{2}(t-s)} H(U)(s) d s\right] \geq 0 .
\end{aligned}
$$

Set

$$
\begin{gathered}
L=\max \left\{\begin{array}{c}
\max \left|\partial_{11} g(u, v)\right|, \max \left|\partial_{2} g(u, v) S^{\prime \prime}(w)\right|+\max \left|\partial_{12} g(u, v) S^{\prime}(w)\right|, \\
\max \left|\partial_{2} g(u, v) S^{\prime \prime}(w)\right|+\max \left|\partial_{21} g(u, v) S^{\prime}(w)\right|, \max \left|\partial_{22} g(u, v) S^{\prime 2}(w)\right|,, \\
u, w \in[0,1], v \in[S(0), S(1)]
\end{array}\right\}, \\
\varpi_{1}=\partial_{1} g(1, S(1))+\partial_{2} g(1, S(1)) S^{\prime}(1)<0
\end{gathered}
$$

and

$$
\varpi_{2}=\partial_{1} g(1, S(1))-\partial_{2} g(1, S(1)) S^{\prime}(1)<0 .
$$

Since $\lim _{t \rightarrow+\infty} U(t)=0$ and $\lim _{t \rightarrow+\infty} V(t)=0$, there exists $t^{\prime}>0$ such that for any $t>t^{\prime}$,

$$
-\frac{1}{4} \varpi_{1}[U(t)+V(t)]>L\left[(1+2 G(2 \beta)) U^{2}(t)+2 U(t) V(t)+V^{2}(t)\right] .
$$

Then by Taylor's expansion, for any $t>t^{\prime}$,

$$
\begin{aligned}
d U^{\prime \prime}(t) & -c U^{\prime}(t) \\
= & g(1-U(t),(h * S(1-U))(t)) \\
\geq & -\partial_{1} g(1, S(1)) U(t)-\partial_{2} g(1, S(1)) S^{\prime}(1) V(t) \\
& -L\left[(1+2 G(2 \beta)) U^{2}(t)+2 U(t) V(t)+V^{2}(t)\right] \\
= & -\frac{1}{4} \varpi_{1} U(t)+\frac{1}{2} \varpi_{2}(V(t)-U(t))-\frac{1}{4} \varpi_{1} V(t)-\frac{1}{4} \varpi_{1}(U(t)+V(t)) \\
& -L\left[(1+2 G(2 \beta)) U^{2}(t)+2 U(t) V(t)+V^{2}(t)\right] \\
\geq & -\frac{1}{4} \varpi_{1} U(t)+\frac{1}{2} \varpi_{2}(V(t)-U(t))-\frac{1}{4} \varpi_{1} V(t) .
\end{aligned}
$$

Now we show that for any $t \in \mathbb{R}, U(t)$ is integrable on $[t,+\infty)$ and there exists $\rho>0$ such that $\sup _{t \in \mathbb{R}} U(t) e^{\rho t}<+\infty$. By Fubini's theorem and Lebesgue's dominated 
convergence theorem, we have, as $y \rightarrow+\infty$,

$$
\begin{aligned}
& \int_{t}^{y}(V(s)-U(s)) d s \\
= & \int_{t}^{y} \int_{0}^{\tau} \int_{-\infty}^{\infty} h(z, r)(U(s-c r-z)-U(s)) d z d r d s \\
= & -\int_{t}^{y} \int_{0}^{\tau} \int_{-\infty}^{\infty}(z+c r) h(z, r) \int_{0}^{1} U^{\prime}(s-\theta(c r+z)) d \theta d z d r d s \\
= & -\int_{0}^{\tau} \int_{-\infty}^{\infty}(z+c r) h(z, r) \int_{0}^{1} \int_{t}^{y} U^{\prime}(s-\theta(c r+z)) d s d \theta d z d r \\
= & -\int_{0}^{\tau} \int_{-\infty}^{\infty}(z+c r) h(z, r) \int_{0}^{1}[U(y-\theta(c r+z))-U(t-\theta(c r+z))] d \theta d z d r \\
\rightarrow & \int_{0}^{\tau} \int_{-\infty}^{\infty}(z+c r) h(z, r) \int_{0}^{1} U(t-\theta(c r+z)) d \theta d z d r .
\end{aligned}
$$

Since $\lim _{t \rightarrow+\infty} U^{\prime}(t)=0$ by Lemma 3.4, integrating (3.1) from $t$ to $+\infty$, we have that for any $t>t^{\prime}$,

$$
\begin{gathered}
-d U^{\prime}(t)+c U(t)-\frac{1}{2} \varpi_{2} \int_{0}^{\tau} \int_{-\infty}^{\infty}(z+c r) h(z, r) \int_{0}^{1} U(t-\theta(c r+z)) d \theta d z d r \\
\geq-\frac{1}{4} \varpi_{1} \int_{t}^{+\infty} U(s) d s-\frac{1}{4} \varpi_{1} \int_{t}^{+\infty} V(s) d s,
\end{gathered}
$$

which implies that $U(t)$ and $V(t)$ are integrable on $[t,+\infty)$.

Now we define a function $W(t)=\int_{t}^{+\infty} U(s) d s$, which is decreasing and satisfies $\lim _{t \rightarrow+\infty} W(t)=0$ and $W(t) \leq W(0)-t$ for $t \leq 0$. Obviously,

$$
\begin{aligned}
\int_{t}^{+\infty} V(s) d s & =\int_{t}^{+\infty} \int_{0}^{\tau} \int_{-\infty}^{\infty} h(z, r) U(s-c r-z) d z d r d s \\
& =\lim _{y \rightarrow+\infty} \int_{t}^{y} \int_{0}^{\tau} \int_{-\infty}^{\infty} h(z, r) U(s-c r-z) d z d r d s \\
& =\lim _{y \rightarrow+\infty} \int_{0}^{\tau} \int_{-\infty}^{\infty} h(z, r) \int_{t}^{y} U(s-c r-z) d s d z d r \\
& =\int_{0}^{\tau} \int_{-\infty}^{\infty} h(z, r) \int_{t}^{+\infty} U(s-c r-z) d s d z d r \\
& =\int_{0}^{\tau} \int_{-\infty}^{\infty} h(z, r) W(t-c r-z) d z d r .
\end{aligned}
$$

Integrating (3.1) from $t$ to $+\infty$ with $t>t^{\prime}$, we get

$$
\begin{aligned}
& -d U^{\prime}(t)+c U(t) \\
& \geq-\frac{1}{4} \varpi_{1} W(t)+\frac{1}{2} \varpi_{2}\left[\int_{0}^{\tau} \int_{-\infty}^{\infty} h(z, r) W(t-c r-z) d z d r-W(t)\right] \\
& \quad-\frac{1}{4} \varpi_{1} \int_{0}^{\tau} \int_{-\infty}^{\infty} h(z, r) W(t-c r-z) d z d r .
\end{aligned}
$$


Note that

$$
\begin{aligned}
& \int_{t}^{y} \int_{0}^{\tau} \int_{-\infty}^{\infty} h(z, r)[W(s-c r-z)-W(s)] d z d r d s \\
& =-\int_{t}^{y} \int_{0}^{\tau} \int_{-\infty}^{\infty}(c r+z) h(z, r) \int_{0}^{1} W^{\prime}(s-\theta(c r+z)) d \theta d z d r d s \\
& =-\int_{0}^{\tau} \int_{-\infty}^{\infty}(c r+z) h(z, r) \int_{0}^{1}[W(y-\theta(c r+z))-W(t-\theta(c r+z))] d \theta d z d r \\
& \rightarrow \int_{0}^{\tau} \int_{-\infty}^{\infty}(c r+z) h(z, r) \int_{0}^{1} W(t-\theta(c r+z)) d \theta d z d r \text { as } y \rightarrow+\infty .
\end{aligned}
$$

Then, for any $t>t^{\prime}$, (3.2) implies that

$$
\begin{aligned}
& d U(t)+c W(t)-\frac{1}{2} \varpi_{2} \int_{0}^{\tau} \int_{-\infty}^{\infty}(c r+z) h(z, r) \int_{0}^{1} W(t-\theta(c r+z)) d \theta d z d r \\
& \geq-\frac{1}{4} \varpi_{1} \int_{t}^{+\infty} W(s) d s-\frac{1}{4} \varpi_{1} \int_{t}^{+\infty} \int_{0}^{\tau} \int_{-\infty}^{\infty} h(z, r) W(s-c r-z) d z d r d s
\end{aligned}
$$

which means that $W(t)$ and $\int_{0}^{\tau} \int_{-\infty}^{\infty} h(z, r) W(t-c r-z) d z d r$ are integrable on $[t,+\infty)$.

Since $W(t)$ is decreasing, then for any $t \in \mathbb{R}$, we have

$$
(z+c r) h(z, r) W(t-(z+c r)) \geq(z+c r) h(z, r) \int_{0}^{1} W(t-\theta(z+c r)) d \theta .
$$

Again, for $z+c r \geq 0$,

$$
\begin{aligned}
W(t-(z+c r)) & =W(t)+\int_{t-(z+c r)}^{t} U(s) d s \leq W(t)+\int_{t-(z+c r)}^{t} U(t) e^{\beta(t-s)} d s \\
& \leq W(t)+\frac{1}{\beta} e^{\beta(z+c r)} U(t) .
\end{aligned}
$$

Consequently, by (3.3), we have

$$
\begin{aligned}
& d U(t)+c W(t)-\frac{1}{2} \varpi_{2} W(t) \int_{0}^{\tau} \int_{-c r}^{\infty}(c r+z) h(z, r) d z d r \\
& -\frac{1}{2 \beta} \varpi_{2} U(t) \int_{0}^{\tau} \int_{-c r}^{\infty}(c r+z) e^{\beta(z+c r)} h(z, r) d z d r \\
& \geq d U(t)+c W(t)-\frac{1}{2} \varpi_{2} \int_{0}^{\tau} \int_{-c r}^{\infty}(c r+z) h(z, r) W(t-(c r+z)) d z d r \\
& \geq d U(t)+c W(t)-\frac{1}{2} \varpi_{2} \int_{0}^{\tau} \int_{-\infty}^{\infty}(c r+z) h(z, r) \int_{0}^{1} W(t-\theta(c r+z)) d \theta d z d r \\
& \geq-\frac{1}{4} \varpi_{1} \int_{t}^{+\infty} W(s) d s .
\end{aligned}
$$

Thus, there exists a sufficiently large $K>0$ such that for any $t>t^{\prime}$ and any $p>0$, $K[U(t)+W(t)] \geq-\frac{1}{4} \varpi_{1} \int_{t}^{+\infty}[U(s)+W(s)] d s \geq-\frac{p}{4} \varpi_{1}[U(t+p)+W(t+p)]$. 
Choosing $p_{0}>0$ sufficiently large, then there exists $\theta_{0} \in(0,1)$ such that for any $t>t^{\prime}, \theta_{0}[U(t)+W(t)] \geq U\left(t+p_{0}\right)+W\left(t+p_{0}\right)$. Let $e(t)=[U(t)+W(t)] e^{\rho t}$, where $\rho=\frac{1}{p_{0}} \ln \frac{1}{\theta_{0}}>0$. Then $e\left(t+p_{0}\right)=\left[U\left(t+p_{0}\right)+W\left(t+p_{0}\right)\right] e^{\rho\left(t+p_{0}\right)} \leq$ $\theta_{0}[U(t)+W(t)] e^{\rho\left(t+p_{0}\right)}=e(t)$. In view of $\lim _{t \rightarrow-\infty}[U(t)+W(t)] e^{\rho t}=0$, then $\sup _{t \in \mathbb{R}}\left\{[U(t)+W(t)] e^{\rho t}\right\}<\infty$, which implies that $\sup _{t \in \mathbb{R}}\left\{U(t) e^{\rho t}\right\}<\infty$.

Next we prove that $\lim _{t \rightarrow+\infty} e^{-\lambda_{11} t} U(t)$ exists. For $\lambda$ with $-\rho<\operatorname{Re} \lambda<0$, we define a two-sided Laplace transform of $U$ by

$$
\ell(\lambda) \equiv \int_{-\infty}^{\infty} e^{-\lambda t} U(t) d t
$$

Note that for $t \leq 0, h(y, r) U(t-c r-y) e^{-\operatorname{Re} \lambda t}<h(y, r) e^{-\operatorname{Re} \lambda t}$ and for $t>0$,

$$
\begin{aligned}
h(y, r) U(t-c r-y) e^{-\operatorname{Re} \lambda t} & =h(y, r) U(t-c r-y) e^{\rho(t-c r-y)} e^{\rho c r} e^{\rho y} e^{(-\rho-\operatorname{Re} \lambda) t} \\
& \leq \widetilde{M} h(y, r) e^{\rho c r} e^{\rho y} e^{(-\rho-\operatorname{Re} \lambda) t},
\end{aligned}
$$

where $\widetilde{M}=\sup _{t \in \mathbb{R}}\left\{U(t) e^{\rho t}\right\}$; then $h(y, r) U(t-c r-y) e^{-\operatorname{Re} \lambda t}$ is integrable on $(r, y, t) \in[0, \tau] \times \mathbb{R} \times \mathbb{R}$. Since $e^{-i \operatorname{Im} \lambda t}$ is bounded and hence $h(y, r) U(t-c r-y) e^{-\lambda t}$ is integrable on $(r, y, t) \in[0, \tau] \times \mathbb{R} \times \mathbb{R}$, by Fubini's Theorem, we have

$$
\begin{aligned}
\int_{-\infty}^{\infty} e^{-\lambda t} V(t) d t & =\int_{-\infty}^{\infty} e^{-\lambda t} \int_{0}^{\tau} \int_{-\infty}^{\infty} h(y, r) U(t-c r-y) d y d r d t \\
& =\int_{0}^{\tau} \int_{-\infty}^{\infty} h(y, r) e^{-\lambda(c r+y)} \int_{-\infty}^{\infty} e^{-\lambda(t-c r-y)} U(t-c r-y) d t d y d r \\
& =\ell(\lambda) \int_{0}^{\tau} \int_{-\infty}^{\infty} h(y, r) e^{-\lambda(c r+y)} d y d r \\
& =\ell(\lambda) G(\lambda) .
\end{aligned}
$$

Since

$$
\begin{aligned}
d U^{\prime \prime}(t)-c U^{\prime}(t) & +\partial_{1} g(1, S(1)) U(t) \\
& +\partial_{2} g(1, S(1)) S^{\prime}(1) V(t) \\
=\partial_{1} g(1, S(1)) & U(t)+\partial_{2} g(1, S(1)) S^{\prime}(1) V(t) \\
& +\partial_{2} g(1, S(1)) S^{\prime}(1) V(t) \\
+ & g(1-U(t),(h * S(1-U))(t))
\end{aligned}
$$

we have

$$
\begin{aligned}
\Delta_{1}(\lambda) \ell(\lambda)= & \int_{-\infty}^{\infty} e^{-\lambda t}\left[\partial_{1} g(1, S(1)) U(t)+\partial_{2} g(1, S(1)) S^{\prime}(1) V(t)\right. \\
& +g(1-U(t),(h * S(1-U))(t))] d t
\end{aligned}
$$

By $\lim _{t \rightarrow+\infty} U(t)=0$ and $\lim _{t \rightarrow+\infty} V(t)=0$, we have

$$
\begin{aligned}
& \partial_{1} g(1, S(1)) U(t)+\partial_{2} g(1, S(1)) S^{\prime}(1) V(t)+g(1-U(t),(h * S(1-U))(t)) \\
& \quad=O\left(U^{2}(t)+V^{2}(t)\right)
\end{aligned}
$$

as $t \rightarrow+\infty$. Hence, the right-hand side of equality (3.4) is defined for $\lambda$ with $-2 \rho<$ $\operatorname{Re} \lambda<0$. Now we use a property of Laplace transforms (Widder [44, p. 58]). Since 
$U(t)>0$, there exists a real number $\vartheta$ such that $\ell(\lambda)$ is analytic for $\vartheta<\operatorname{Re} \lambda<0$ and $\ell(\lambda)$ has a singularity at $\lambda=\vartheta$. Hence, $\ell(\lambda)$ is defined for $\operatorname{Re} \lambda>\lambda_{11}$.

We rewrite (3.4) as

$$
\begin{aligned}
\int_{0}^{+\infty} U(t) e^{-\lambda t} d t & =-\int_{-\infty}^{0} U(t) e^{-\lambda t} d t+\frac{1}{\Delta_{1}(\lambda)} \int_{-\infty}^{\infty} e^{-\lambda t}\left[\partial_{1} g(1, S(1)) U(t)\right. \\
& \left.+\partial_{2} g(1, S(1)) S^{\prime}(1) V(t)+g(1-U(t),(h * S(1-U))(t))\right] d t .
\end{aligned}
$$

Note that $\int_{-\infty}^{0} U(t) e^{-\lambda t} d t$ is analytic for $\operatorname{Re} \lambda<0$. Also, the equation $\Delta_{1}(\lambda)=0$ does not have any zero with $\operatorname{Re} \lambda=\lambda_{11}$ other than $\lambda=\lambda_{11}$. In fact, let $\lambda=\lambda_{11}+i \gamma$; then $\Delta_{1}(\lambda)=0$ implies

$-\partial_{2} g(1, S(1)) S^{\prime}(1) \int_{0}^{\tau} \int_{-\infty}^{\infty} h(y, r) e^{-\lambda_{11}(y+c r)}[\cos \gamma c r \cos \gamma y-\sin \gamma c r \sin \gamma y] d y d r$

$$
=d \lambda_{11}^{2}-d \gamma^{2}-c \lambda_{11}+\partial_{1} g(1, S(1))
$$

and

$$
\begin{aligned}
& 2 d \gamma-c \gamma-\partial_{2} g(1, S(1)) S^{\prime}(1) \\
& \quad \times \int_{0}^{\tau} \int_{-\infty}^{\infty} h(y, r) e^{-\lambda_{11}(y+c r)}[\sin \gamma c r \cos \gamma y+\cos \gamma c r \sin \gamma y] d y d r=0
\end{aligned}
$$

By using $\Delta_{1}\left(\lambda_{11}\right)=0$, then (3.5) can be rewritten as

$$
\begin{aligned}
-d \gamma^{2}= & \partial_{2} g(1, S(1)) S^{\prime}(1) \int_{0}^{\tau} \int_{-\infty}^{\infty} h(y, r) e^{-\lambda_{11}(y+c r)}\left[2\left(\sin \frac{\gamma c r}{2}\right)^{2}+2\left(\sin \frac{\gamma y}{2}\right)^{2}\right. \\
& \left.-4\left(\sin \frac{\gamma c r}{2}\right)^{2}\left(\sin \frac{\gamma y}{2}\right)^{2}+\sin \gamma c r \sin \gamma y\right] d y d r .
\end{aligned}
$$

Since

$$
\begin{aligned}
& 2\left(\sin \frac{\gamma c r}{2}\right)^{2}+2\left(\sin \frac{\gamma y}{2}\right)^{2}-4\left(\sin \frac{\gamma c r}{2}\right)^{2}\left(\sin \frac{\gamma y}{2}\right)^{2}+\sin \gamma c r \sin \gamma y \\
& \quad=2\left(\sin \frac{\gamma c r}{2}\right)^{2}\left(\cos \frac{\gamma y}{2}\right)^{2}+2\left(\cos \frac{\gamma c r}{2}\right)^{2}\left(\sin \frac{\gamma y}{2}\right)^{2}+\sin \gamma c r \sin \gamma y \\
& \quad \geq 4\left|\sin \frac{\gamma c r}{2} \cos \frac{\gamma c r}{2} \sin \frac{\gamma y}{2} \cos \frac{\gamma y}{2}\right|+\sin \gamma c r \sin \gamma y \\
& \quad=|\sin \gamma c r \sin \gamma y|+\sin \gamma c r \sin \gamma y \geq 0,
\end{aligned}
$$

we have $-d \gamma^{2} \geq 0$, which implies $\gamma=0$.

Since $U(t)$ is decreasing, then Lemma 3.3 implies that $\lim _{t \rightarrow \infty} e^{-\lambda_{11} t}(1-\phi(t))=$ $\lim _{t \rightarrow \infty} e^{-\lambda_{11} t} U(t)$ exists. Take $\lim _{t \rightarrow \infty} e^{-\lambda_{11} t}(1-\phi(t))=a_{11}$. We now prove that $\lim _{t \rightarrow \infty} e^{-\lambda_{11} t} \phi^{\prime}(t)=-\lambda_{11} a_{11}$. From Lebesgue's dominated convergence theorem, we know that

$$
\begin{aligned}
\lim _{t \rightarrow \infty} e^{-\lambda_{11} t} V(t) & \\
& =\int_{0}^{\tau} \int_{-\infty}^{\infty} h(z, r) e^{-\lambda_{11}(z+c r)}\left[\lim _{t \rightarrow \infty} e^{-\lambda_{11}(t-z-c r)} U(t-z-c r)\right] d z d r \\
& =a_{11} \int_{0}^{\tau} \int_{-\infty}^{\infty} h(z, r) e^{-\lambda_{11}(z+c r)} d z d r=a_{11} G\left(\lambda_{11}\right) .
\end{aligned}
$$


Since as $t \rightarrow+\infty$,

$$
\begin{aligned}
& g(1-U(t),(h * S(1-U))(t)) \\
& \quad=-\partial_{1} g(1, S(1)) U(t)-\partial_{2} g(1, S(1)) S^{\prime}(1) V(t)+O\left(U^{2}(t)+V^{2}(t)\right),
\end{aligned}
$$

then

$$
\begin{aligned}
& \lim _{t \rightarrow \infty} e^{-\lambda_{11} t} g(1-U(t),(h * S(1-U))(t)) \\
& \quad=-a_{11}\left[\partial_{1} g(1, S(1))+\partial_{2} g(1, S(1)) S^{\prime}(1) G\left(\lambda_{11}\right)\right] .
\end{aligned}
$$

Using $\lim _{t \rightarrow \infty} U^{\prime}(t)=0$ and integrating the two sides of the equality $d U^{\prime \prime}(t)=$ $c U^{\prime}(t)+g(1-U(t),(h * S(1-U))(t))$ from $t$ to $+\infty$, we have

$$
d U^{\prime}(t)=c U(t)-\int_{t}^{\infty} g(1-U(s),(h * S(1-U))(s)) d t .
$$

Thus,

$$
\begin{aligned}
\lim _{t \rightarrow \infty} e^{-\lambda_{11} t} \phi^{\prime}(t) & =-\lim _{t \rightarrow \infty} e^{-\lambda_{11} t} U^{\prime}(t) \\
& =-\frac{c a_{11}}{d}+\frac{1}{d} \lim _{t \rightarrow \infty} e^{-\lambda_{11} t} \int_{t}^{\infty} g(1-U(s),(h * S(1-U))(s)) d t \\
& =-\frac{c}{d}-\frac{\lim _{t \rightarrow \infty} e^{-\lambda_{11} t} g(1-U(t),(h * S(1-U))(t))}{d \lambda_{11}} \\
& =-\frac{a_{11}\left[c \lambda_{11}-\partial_{1} g(1, S(1))-\partial_{2} g(1, S(1)) S^{\prime}(1) G\left(\lambda_{11}\right)\right]}{d \lambda_{11}} \\
& =-a_{11} \lambda_{11} .
\end{aligned}
$$

We have completed the proof of the first conclusion. The remainder can be proved by similar arguments. The proof is complete.

\section{EXISTENCE OF ENTIRE SOlutions}

We study the following ordinary differential equation:

$$
\frac{d}{d t} p(t)=c+N e^{\alpha p(t)}, \quad t \leq 0,
$$

where $N, c$ and $\alpha$ are positive constants. Solving this equation explicitly, we have

$$
p(t)=p(0)+c t-\frac{1}{\alpha} \ln \left\{1+\frac{N}{c} e^{\alpha p(0)}\left(1-e^{c \alpha t}\right)\right\} .
$$

It is clear that the solution $p(t)$ is monotone increasing. Let

$$
\omega=p(0)-\frac{1}{\alpha} \ln \left\{1+\frac{N}{c} e^{\alpha p(0)}\right\} .
$$

Then from the identity $p(t)-c t-\omega=-\frac{1}{\alpha} \ln \left\{1-r e^{c \alpha t} /(1+r)\right\}$ and $r=N e^{\alpha p(0)} / c$, it follows that for some positive constant $R_{0}$,

$$
0<p(t)-c t-\omega \leq R_{0} e^{c \alpha t}, \forall t \leq 0 .
$$

We note that the above argument about $p(t)$ was first given by Guo and Morita [20] (see also Fukao et al. [16]). In the sequel of this section, we always assume 
that (1.1) has an increasing traveling wave solution $\phi$ with wave speed $c>0$. By Theorem 3.5, there are positive constants $k, K, \mu, \eta$ such that

$$
\begin{aligned}
& k e^{\lambda_{02} t} \leq \phi(t) \leq K e^{\lambda_{02} t}, k e^{\lambda_{02} t} \leq(h * \phi)(t) \leq K e^{\lambda_{02} t}(t \leq 0), \\
& \eta k e^{\lambda_{02} t} \leq \eta \phi(t) \leq \phi^{\prime}(t), \eta k e^{\lambda_{02} t} \leq \eta(h * \phi)(t) \leq \phi^{\prime}(t) \quad(t \leq 0),
\end{aligned}
$$

$\eta \mu e^{\lambda_{11} t} \leq \eta(1-\phi(t)) \leq \phi^{\prime}(t), \eta \mu e^{\lambda_{11} t} \leq \eta(1-(h * \phi)(t)) \leq \phi^{\prime}(t) \quad(t \geq 0)$.

Lemma 4.1. There exists $T<0$ such that $\bar{u}(x, t)$ defined by

$$
\bar{u}(x, t)=\min \{\phi(x+p(t))+\phi(-x+p(t)), 1\}
$$

is a supersolution of (1.1) on $(-\infty, T) . p(t)$ is defined by (4.1) with $\alpha=\lambda_{02}$, $p(0) \leq 0$ and

$$
N \geq \max \left\{\frac{2 L K^{2}}{\eta k}, \frac{4 L K}{\eta \mu}, \frac{4\left(L+\frac{1}{2}+\int_{0}^{\tau} \int_{0}^{\infty} h(y, r) e^{2 \beta y} d y d r\right) K}{\eta}\right\},
$$

where $L$ and $\beta$ are defined in Theorem 3.5.

Proof. Define

$$
\begin{aligned}
& A_{1}^{+}=\left\{(x, t) \in \mathbb{R}^{2}: \phi(x+p(t))+\phi(-x+p(t))>1\right\} \\
& A_{1}^{-}=\left\{(x, t) \in \mathbb{R}^{2}: \phi(x+p(t))+\phi(-x+p(t))<1\right\} .
\end{aligned}
$$

If $(x, t) \in A_{1}^{+}$, then $\bar{u}(x, t)=1$ and

$$
\begin{aligned}
\frac{\partial \bar{u}}{\partial t}-d \Delta \bar{u}-g(\bar{u}(x, t),(h * S(\bar{u}))(x, t)) & =-g(1,(h * S(\bar{u}))(x, t)) \\
& \geq-g(1, S(1))=0 .
\end{aligned}
$$

Now we consider the case $(x, t) \in A_{1}^{-}$. In this case, $\bar{u}(x, t)=\phi(x+p(t))+$ $\phi(-x+p(t))$. Consequently,

$$
\begin{aligned}
& \frac{\partial \bar{u}}{\partial t}-d \Delta \bar{u}-g(\bar{u}(x, t),(h * S(\bar{u}))(x, t)) \\
= & p^{\prime}(t)\left[\phi^{\prime}(x+p)+\phi^{\prime}(-x+p)\right]-d\left[\phi^{\prime \prime}(x+p)+\phi^{\prime \prime}(-x+p)\right] \\
& -g(\phi(x+p)+\phi(-x+p),(h * S(\bar{u}))(x, t)) \\
= & {\left[p^{\prime}(t)-c\right]\left[\phi^{\prime}(x+p)+\phi^{\prime}(-x+p)\right]+g(\phi(x+p),(h * S(\phi))(x+p)) } \\
& +g(\phi(-x+p),(h * S(\phi))(-x+p)) \\
& -g(\phi(x+p)+\phi(-x+p),(h * S(\bar{u}))(x, t)) \\
= & {\left[\phi^{\prime}(x+p)+\phi^{\prime}(-x+p)\right] N e^{\lambda_{02} p}-R(x, t), }
\end{aligned}
$$

where

$$
\begin{aligned}
R(x, t)= & g(\phi(x+p)+\phi(-x+p),(h * S(\bar{u}))(x, t)) \\
& -g(\phi(x+p),(h * S(\phi))(x+p))-g(\phi(-x+p),(h * S(\phi))(-x+p)) .
\end{aligned}
$$


Since for $r \geq 0$,

$$
\begin{aligned}
& p(t-r) \\
= & p(0)+c(t-r)-\frac{1}{\lambda_{02}} \ln \left\{1+\frac{N}{c} e^{\lambda_{02} p(0)}\left(1-e^{c \lambda_{02}(t-r)}\right)\right\} \\
= & p(t)-c r \\
& +\frac{1}{\lambda_{02}} \ln \left\{\frac{1+\frac{N}{c} e^{\lambda_{02} p(0)}\left(1-e^{c \lambda_{02} t}\right)}{1+\frac{N}{c} e^{\lambda_{02} p(0)}\left(1-e^{c \lambda_{02} t}\right)+\frac{N}{c} e^{\lambda_{02} p(0)} e^{c \lambda_{02} t}\left(1-e^{-c \lambda_{02} r}\right)}\right\} \\
\leq & p(t)-c r,
\end{aligned}
$$

it follows that

$$
\begin{aligned}
\xi(y, r) & =\bar{u}(x-y, t-r)-\phi(x+p(t)-y-c r) \\
& \leq \phi(x-y+p(t-r))+\phi(-x+y+p(t-r))-\phi(x+p(t)-y-c r) \\
& \leq \phi(x-y+p(t)-c r)+\phi(-x+y+p(t)-c r)-\phi(x+p(t)-y-c r) \\
& =\phi(-x+y+p(t)-c r) .
\end{aligned}
$$

Consequently,

$$
\begin{aligned}
& R(x, t) \\
\leq \quad & g(\phi(x+p)+\phi(-x+p),(h * S(\bar{u}))(x, t))-g(\phi(x+p),(h * S(\phi))(x+p)) \\
& -g\left(\phi(-x+p), \int_{0}^{\tau} \int_{-\infty}^{\infty} h(y, r) S(\xi(y, r)) d y d r\right) \\
= & \int_{0}^{1}\left[\partial_{1} g(\phi(x+p)+\theta \phi(-x+p), \zeta(x, t)) \phi(-x+p)\right. \\
& +\partial_{2} g(\phi(x+p)+\theta \phi(-x+p), \zeta(x, t)) \\
& \left.\times \int_{0}^{\tau} \int_{-\infty}^{\infty} h(y, r) S^{\prime}(\phi(x+p-y-c r)+\theta \xi(y, r)) \xi(y, r) d y d r\right] d \theta \\
& -\int_{0}^{1}\left[\partial_{1} g\left(\theta \phi(-x+p), \int_{0}^{\tau} \int_{-\infty}^{\infty} h(y, r) S(\theta \xi(y, r)) d y d r\right) \phi(-x+p)\right. \\
& +\partial_{2} g\left(\theta \phi(-x+p), \int_{0}^{\tau} \int_{-\infty}^{\infty} h(y, r) S(\theta \xi(y, r)) d y d r\right) \\
& \left.\times \int_{0}^{\tau} \int_{-\infty}^{\infty} h(y, r) S^{\prime}(\theta \xi(y, r)) \xi(y, r) d y d r\right] d \theta \\
\leq & L[\phi(x+p) \phi(-x+p)+\phi(-x+p)(h * \phi)(x+p) \\
& +\phi(x+p) \int_{0}^{\tau} \int_{-\infty}^{\infty} h(y, r) \xi(y, r) d y d r \\
& \left.+(h * \phi)(x+p) \int_{0}^{\tau} \int_{-\infty}^{\infty} h(y, r) \xi(y, r) d y d r\right] \\
\leq & L[\phi(x+p) \phi(-x+p)+\phi(-x+p)(h * \phi)(x+p) \\
& +\phi(x+p)(h * \phi)(-x+p)+(h * \phi)(x+p)(h * \phi)(-x+p)],
\end{aligned}
$$

where $\zeta(x, t)=\int_{0}^{\tau} \int_{-\infty}^{\infty} h(y, r) S(\phi(x+p(t)-y-c r)+\theta \xi(y, r)) d y d r$.

Note that $p(t)<0$ for all $t \leq 0$. Let $U(x, t)=\frac{R(x, t)}{\phi^{\prime}(x+p(t))+\phi^{\prime}(-x+p(t))}$. Now we estimate $U(x, t)$. 
Case I: $\lambda_{02} \geq-\lambda_{11}$. We divide $\mathbb{R}$ into 3 regions.

(i) $p(t) \leq x \leq-p(t)$. By (4.4), we have the estimate $R(x, t) \leq 4 L K^{2} e^{2 \lambda_{02} p}$. Also, by (4.5), we have

$$
\begin{aligned}
& \phi^{\prime}(x+p)+\phi^{\prime}(-x+p) \geq \eta[\phi(x+p)+\phi(-x+p)] \\
\geq & \eta k\left[e^{\lambda_{02}(x+p)}+e^{\lambda_{02}(-x+p)}\right]=\eta k\left(e^{\lambda_{02} x}+e^{-\lambda_{02} x}\right) e^{\lambda_{02} p} \geq 2 \eta k e^{\lambda_{02} p} .
\end{aligned}
$$

Hence, we have

$$
U(x, t) \leq \frac{2 L K^{2}}{\eta k} e^{\lambda_{02} p}
$$

(ii) $x \leq p(t)$. It follows from (4.6) that

$$
\begin{aligned}
U(x, t) & \leq \frac{2 L(\phi(x+p)+(h * \phi)(x+p))}{\phi^{\prime}(-x+p(t))} \leq \frac{4 L K e^{\lambda_{02}(x+p)}}{\eta \mu e^{\lambda_{11}(-x+p)}} \\
& =\frac{4 L K e^{\lambda_{02} p}}{\eta \mu e^{\left(-\lambda_{11}-\lambda_{02}\right) x} e^{\lambda_{11} p}} \leq \frac{4 L K}{\eta \mu} e^{\lambda_{02} p}
\end{aligned}
$$

(iii) $x \geq-p(t)$. By the symmetry $U(-x, t)=U(x, t)$ and (4.8), we obtain

$$
U(x, t) \leq \frac{4 L K}{\eta \mu} e^{\lambda_{02} p}
$$

Thus, combining (4.7)-(4.9) yields $\frac{\partial \bar{u}}{\partial t}-d \Delta \bar{u}-g(\bar{u}(x, t),(h * S(\bar{u}))(x, t)) \geq 0$.

Case II: $0<\lambda_{02}<-\lambda_{11}$. In this case, since $\lambda_{02}$ and $\lambda_{11}$ satisfy

$$
\begin{aligned}
& d \lambda_{02}^{2}-c \lambda_{02}+\partial_{1} g(0, S(0))+\partial_{2} g(0, S(0)) S^{\prime}(0) G\left(\lambda_{02}\right)=0 \\
& d \lambda_{11}^{2}-c \lambda_{11}+\partial_{1} g(1, S(1))+\partial_{2} g(1, S(1)) S^{\prime}(1) G\left(\lambda_{11}\right)=0
\end{aligned}
$$

and $G\left(\lambda_{02}\right)<G\left(\lambda_{11}\right)$, then

$$
\begin{aligned}
& \partial_{1} g(0, S(0))+\partial_{2} g(0, S(0)) S^{\prime}(0) G\left(\lambda_{02}\right) \\
& \quad>\partial_{1} g(1, S(1))+\partial_{2} g(1, S(1)) S^{\prime}(1) G\left(\lambda_{02}\right)
\end{aligned}
$$

Set

$$
\begin{aligned}
\kappa= & \partial_{1} g(0, S(0))+\partial_{2} g(0, S(0)) S^{\prime}(0) G\left(\lambda_{02}\right) \\
& -\partial_{1} g(1, S(1))+\partial_{2} g(1, S(1)) S^{\prime}(1) G\left(\lambda_{02}\right) .
\end{aligned}
$$

Then there exists $\delta>0$ with $\delta \leq S(1)-S(0)$ such that

$$
\partial_{1} g(u, v)+\partial_{2} g(u, v) w \varpi<\partial_{1} g(0, S(0))+\partial_{2} g(0, S(0)) S^{\prime}(0) G\left(\lambda_{02}\right)-\frac{\kappa}{2}
$$

for any $u \in(1-\delta, 1), v \in(S(1)-\delta, S(1)), w \in\left(0, S^{\prime}(1)+\delta\right)$ and $\varpi \in\left(0, G\left(\lambda_{02}\right)+\delta\right)$.

Take $B>c \tau$ such that

$$
\left[\int_{0}^{\tau} \int_{-\infty}^{-B} h(y, r) d y d r+\int_{0}^{\tau} \int_{B}^{+\infty} h(y, r) e^{\beta(y-c r)} d y d r\right] \max _{u \in[0,1]} S^{\prime}(u) \leq \frac{\delta}{2} G\left(\lambda_{02}\right)
$$


where $\beta=\max \left\{-\lambda_{1}, \lambda_{2}\right\}$ is defined in Theorem 3.5. As in the proof of Theorem 3.5. we can show that $\phi(t) e^{-\beta t}$ is decreasing. Thus, we have

$$
\begin{aligned}
& \int_{0}^{\tau} \int_{-\infty}^{-B}+\int_{0}^{\tau} \int_{B}^{\infty} h(y, r) S^{\prime}(\phi(x+p(t)-y-c r)+\theta \xi(y, r)) \xi(y, r) d y d r \\
& \leq\left[\int_{0}^{\tau} \int_{-\infty}^{-B}+\int_{0}^{\tau} \int_{B}^{\infty} h(y, r) \phi(-x+p(t)+y-c r) d y d r\right] \max _{u \in[0,1]} S^{\prime}(u) \\
& \leq\left[\int_{0}^{\tau} \int_{-\infty}^{-B} h(y, r) d y d r+\int_{0}^{\tau} \int_{B}^{\infty} h(y, r) e^{\beta(y-c r)} d y d r\right] \max _{u \in[0,1]} S^{\prime}(u) \phi(-x+p(t)) \\
& \leq \frac{\delta}{2} G\left(\lambda_{02}\right) \phi(-x+p(t)) .
\end{aligned}
$$

Since $S^{\prime}(u)$ is continuous on $[0,1]$, then there exists $\rho_{1} \in(0, \delta)$ such that for $u \in$ $\left(1-\rho_{1}, 1\right], S^{\prime}(u) \in\left[0, S^{\prime}(1)+\delta / 2\right]$. Noting that

$$
\lim _{z \rightarrow \infty} \int_{0}^{\tau} \int_{-\infty}^{\infty} h(y, r) S(\phi(z-y-c r)) d y d r=S(1) \quad \text { and } \quad \lim _{z \rightarrow \infty} \phi(z)=1,
$$

we can translate $\phi(z)$ along the $z$-axis so that for any $z \geq-B-c \tau, \phi(z) \in$ $\left(1-\rho_{1}, 1\right]$ and for any $z \geq 0$,

$$
\int_{0}^{\tau} \int_{-\infty}^{\infty} h(y, r) S(\phi(z-y-c r)) d y d r \geq S(1)-\delta .
$$

Hence, $\phi(x+p(t)-y-c r)+\theta \xi(y, r) \in\left(1-\rho_{1}, 1\right]$ for any $x \geq-p(t), y \in[-B, B]$ and $r \in[0, \tau]$. Then, for any $x \geq-p(t), y \in[-B, B]$ and $r \in[0, \tau]$,

$$
\begin{gathered}
S^{\prime}(\phi(x+p-y-c r)+\theta \xi(y, r)) \in\left[0, S^{\prime}(1)+\delta / 2\right] \\
\int_{0}^{\tau} \int_{-\infty}^{\infty} h(y, r) S(\phi(x+p-y-c r)+\theta \xi(y, r)) d y d r \in(S(1)-\delta, S(1)) .
\end{gathered}
$$

In view of

$$
\lim _{z \rightarrow-\infty} \frac{\int_{0}^{\tau} \int_{-B}^{B} h(y, r) \phi(z+y-c r) d y d r}{\phi(z)} \leq \lim _{z \rightarrow-\infty} \frac{(h * \phi)(z)}{\phi(z)}=G\left(\lambda_{02}\right),
$$

we can take $T_{1} \leq 0$ so that for any $t \leq T_{1}$ and $x \geq-p(t)$,

$$
\begin{gathered}
\int_{0}^{\tau} \int_{-\infty}^{\infty} h(y, r) \phi(-x+p(t)+y-c r) d y d r \leq\left(G\left(\lambda_{02}\right)+\delta\right) \phi(-x+p(t)), \\
\left(\partial_{2} g(0, S(0)) S^{\prime}(0) G\left(\lambda_{02}\right)-\frac{\kappa}{2}\right) \phi(-x+p(t)) \\
\leq \partial_{2} g(0, S(0)) S^{\prime}(0) \int_{0}^{\tau} \int_{-\infty}^{\infty} h(y, r) \phi(-x+p(t)+y-c r) d y d r .
\end{gathered}
$$


Thus, by (4.10)-(4.14), for any $t \leq T_{1}$ and $x \geq-p(t)$, we have

$$
\begin{aligned}
& \int_{0}^{1}\left[\partial_{1} g(\phi(x+p(t))+\theta \phi(-x+p(t)), \zeta(x, t)) \phi(-x+p(t))\right. \\
& +\partial_{2} g(\phi(x+p(t))+\theta \phi(-x+p(t)), \zeta(x, t)) \\
& \left.\times \int_{0}^{\tau} \int_{-\infty}^{\infty} h(y, r) S^{\prime}(\phi(x+p(t)-y-c r)+\theta \xi(y, r)) \xi(y, r) d y d r\right] d \theta \\
\leq & {\left[\partial_{1} g(0, S(0))+\partial_{2} g(0, S(0)) S^{\prime}(0) G\left(\lambda_{02}\right)-\frac{\kappa}{2}\right] \phi(-x+p(t)) } \\
\leq & \partial_{1} g(0, S(0)) \phi(-x+p(t))+\partial_{2} g(0, S(0)) S^{\prime}(0)(h * \phi)(-x+p(t)) .
\end{aligned}
$$

Consequently, for any $t \leq T_{1}$ and $x \geq-p(t)$, we have

$$
\begin{aligned}
R(x, t) \leq & \partial_{1} g(0, S(0)) \phi(-x+p)+\partial_{2} g(0, S(0)) S^{\prime}(0)(h * \phi)(-x+p(t)) \\
& -\int_{0}^{1}\left[\partial_{1} g(\theta \phi(-x+p),(h * S(\theta \phi))(-x+p)) \phi(-x+p)\right. \\
& +\partial_{2} g(\theta \phi(-x+p),(h * S(\theta \phi))(-x+p)) \\
& \left.\times \int_{0}^{\tau} \int_{-\infty}^{\infty} h(y, r) S^{\prime}(\theta \phi(-x+p+y-c r)) \phi(-x+p+y-c r) d y d r\right] d \theta \\
\leq & L^{\prime}\left[\phi^{2}(-x+p)+2 \phi(-x+p)(h * \phi)(-x+p)+(h * \phi)^{2}(-x+p)\right],
\end{aligned}
$$

where $L^{\prime}=L+\frac{1}{2}+\int_{0}^{\tau} \int_{0}^{\infty} h(y, r) e^{2 \beta y} d y d r$.

As in the proof of Case I, we divide $\mathbb{R}$ into three intervals $[p,-p],(-\infty, p]$ and $[-p, \infty)$. In the interval $[p,-p]$, we obtain the same estimate as (4.7) for $U(x, t)$. For $x>-p>0$, by (4.15), we obtain

$U(x, t) \leq \frac{L^{\prime}[\phi(-x+p)+(h * \phi)(-x+p)]^{2}}{\phi^{\prime}(-x+p)} \leq \frac{4 L^{\prime} K}{\eta} e^{\lambda_{02}(-x+p)} \leq \frac{4 L^{\prime} K}{\eta} e^{\lambda_{02} p}$.

The estimate for $x \leq p$ can be derived as the case for $x \geq-p$ by the symmetry of $U(x, t)$. Hence, $\frac{\partial \bar{u}}{\partial t}-d \Delta \bar{u}-g(\bar{u}(x, t),(h * S(\bar{u}))(x, t)) \geq 0$.

In order to show that there exists $T \leq 0$ so that $\bar{u}(x, t)$ is a supersolution of (1.1) in $\mathbb{R} \times(-\infty, T)$, we first show the following claim.

Claim. There exists $T \leq 0$ so that for every $t<T$, there are only a finite number of points in $x \in \mathbb{R}$ so that $\phi(x+p(t))+\phi(-x+p(t))=1$.

In fact, if $\lambda_{02}>-\lambda_{11}$, then for sufficiently large $x>-p(t)$,

$$
\phi(x+p(t))+\phi(-x+p(t)) \leq 1-\mu e^{\lambda_{11}(x+p(t))}+K e^{\lambda_{02}(-x+p(t))}<1
$$

and for sufficiently large $|x|$ with $x<p(t)$,

$$
\phi(x+p(t))+\phi(-x+p(t)) \leq 1-\mu e^{\lambda_{11}(-x+p(t))}+K e^{\lambda_{02}(x+p(t))}<1 .
$$

Similarly, we can show that for sufficiently large $|x|>|p(t)|$,

$$
\phi(x+p(t))+\phi(-x+p(t))>1
$$

if $\lambda_{02}<-\lambda_{11}$. If $\lambda_{02}=-\lambda_{11}$, we can take a $T_{2}<0$ so that for $t<T_{2}, \mu e^{-\lambda_{02} p(t)}-$ $K e^{\lambda_{02} p(t)}>0$. Then for sufficiently large $x>-p(t), t<T_{2}$,

$$
\begin{aligned}
\phi(x+p(t))+\phi(-x+p(t)) & \leq 1-\mu e^{\lambda_{11}(x+p(t))}+K e^{\lambda_{02}(-x+p(t))} \\
& =1-e^{-\lambda_{02} x}\left(\mu e^{-\lambda_{02} p(t)}-K e^{\lambda_{02} p(t)}\right)<1 .
\end{aligned}
$$


By the symmetry, for sufficiently large $|x|$ with $x<p(t), t<T_{2}, \phi(x+p(t))+$ $\phi(-x+p(t))<1$. Now choose $T=0$ if $\lambda_{02}>-\lambda_{11}, T=T_{1}$ if $\lambda_{02}<-\lambda_{11}$ and $T=T_{2}$ if $\lambda_{02}=-\lambda_{11}$. Then the claim follows.

So far, for $T \leq 0$ defined in the above claim, we have proved for any $x \in \mathbb{R}$ and $t \in(-\infty, T)$ with $(x, t) \in A_{1}^{+} \cup A_{1}^{-}$,

$$
\frac{\partial \bar{u}}{\partial t}-d \Delta \bar{u}-g(\bar{u}(x, t),(h * S(\bar{u}))(x, t)) \geq 0,
$$

and for every $t<T$, there are only a finite number of points in $x \in \mathbb{R}$ such that $\phi(x+p(t))+\phi(-x+p(t))=1$. In the following, we show that $\bar{u}(x, t)$ is a supersolution of (1.1) in $\mathbb{R} \times(-\infty, T)$. Assume that $x\left(t_{0}\right) \in \mathbb{R}$ satisfies $\phi\left(x\left(t_{0}\right)+p\left(t_{0}\right)\right)+\phi\left(-x\left(t_{0}\right)+p\left(t_{0}\right)\right)=1$ for $t_{0}<T$. It is easy to see that $\frac{\partial}{\partial x} \bar{u}\left(x\left(t_{0}\right)-0, t_{0}\right) \geq \frac{\partial}{\partial x} \bar{u}\left(x\left(t_{0}\right)+0, t_{0}\right)$. By using the inequality (4.16) and a similar argument as in [42] and [43] for the function

$$
\frac{1}{\sqrt{4 \pi d(t-r)}} \int_{-\infty}^{\infty} e^{\frac{-(x-y)^{2}}{4 d(t-r)}} \bar{u}\left(y, T^{\prime}+r\right) d y, \quad 0 \leq r<t \leq T-T^{\prime},
$$

we can show that for every $T^{\prime}<T, w(x, t)=\bar{u}\left(x, t+T^{\prime}\right)$, where $(x, t) \in \mathbb{R} \times$ $\left[-\tau, T-T^{\prime}\right)$, is a supersolution of (1.1) on $\mathbb{R} \times\left[0, T-T^{\prime}\right)$. The proof is complete.

Lemma 4.2. $\underline{u}(x, t)=\max \{\phi(x+c t+\omega), \phi(-x+c t+\omega)\}$ is a subsolution of (1.1) on $\mathbb{R} \times(-\infty, 0)$, where $\omega$ is defined by (4.3).

Proof. When $x>0, v(x, t)=\phi(x+c t+\omega)$, hence,

$$
\begin{aligned}
& \frac{\partial \underline{u}}{\partial t}-d \Delta \underline{u}-g(\underline{u}(x, t),(h * S(\underline{u}))(x, t)) \\
= & c \phi^{\prime}(x+c t+\omega)-d \phi^{\prime \prime}(x+c t+\omega)-g(\phi(x+c t+\omega),(h * S(\underline{u}))(x, t)) \\
= & g(\phi(x+c t+\omega),(h * S(\phi))(x+c t+\omega)) \\
& -g(\phi(x+c t+\omega),(h * S(\underline{u}))(x, t)) \leq 0 .
\end{aligned}
$$

Similarly, we can prove that for $x<0, \frac{\partial \underline{u}}{\partial t}-d \Delta \underline{u}-g(\underline{u}(x, t),(h * S(\underline{u}))(x, t)) \leq 0$.

Note that for every $t<0$,

$$
\frac{\partial}{\partial x} \underline{u}(0+0, t)=\phi^{\prime}(c t+\omega)>-\phi^{\prime}(c t+\omega)=\frac{\partial}{\partial x} \underline{u}(0-0, t) .
$$

Using a similar argument as in 42] and 43, for the function

$$
\frac{1}{\sqrt{4 \pi d(t-r)}} \int_{-\infty}^{\infty} e^{\frac{-(x-y)^{2}}{4 d(t-r)}} \underline{u}\left(y, T^{\prime}+r\right) d y, \quad 0 \leq r<t \leq-T^{\prime},
$$

we can show that for every $T^{\prime}<0, w(x, t)=\underline{u}\left(x, t+T^{\prime}\right)$ defined on $(x, t) \in$ $\mathbb{R} \times\left[-\tau,-T^{\prime}\right)$ is a subsolution of (1.1) on $\mathbb{R} \times\left[0,-T^{\prime}\right)$. The proof is complete.

Proposition 4.3. Suppose that $u(x, t)$ is a solution of (1.1) with initial value $\varphi \in C_{[0,1]}$. Then there exists a positive constant $M>0$ such that for any $\varphi \in C_{[0,1]}$, $x \in \mathbb{R}$ and $t \geq 2(\tau+1),\left|\frac{\partial}{\partial t} u(x, t)\right| \leq M,\left|\frac{\partial}{\partial x} u(x, t)\right| \leq M$ and $\left|\frac{\partial^{2}}{\partial x^{2}} u(x, t)\right| \leq M$, and for any $\varphi \in C_{[0,1]}, x \in \mathbb{R}$ and $t \geq 3(\tau+1),\left|\frac{\partial^{2}}{\partial t^{2}} u(x, t)\right| \leq M,\left|\frac{\partial^{2}}{\partial t \partial x} u(x, t)\right| \leq$ $M,\left|\frac{\partial^{2}}{\partial x \partial t} u(x, t)\right| \leq M,\left|\frac{\partial^{3}}{\partial x^{2} \partial t} u(x, t)\right| \leq M$ and $\left|\frac{\partial^{3}}{\partial x^{3}} u(x, t)\right| \leq M$. 
Proof. First, by comparison, there is $0 \leq u(x, t) \leq 1$ for any $(x, t) \in \mathbb{R} \times[-\tau, \infty)$. Note that for $s \geq \tau$ and $t>s$,

$$
\begin{aligned}
u(x, t)= & \int_{-\infty}^{\infty} \frac{1}{\sqrt{4 \pi d(t-s)}} e^{-\frac{(x-y)^{2}}{4 d(t-s)}} u(y, s) d y \\
& +\int_{s}^{t} \int_{-\infty}^{\infty} \frac{1}{\sqrt{4 \pi d(t-r)}} e^{-\frac{(x-y)^{2}}{4 d(t-r)}} F\left(u_{r}\right) d y d r .
\end{aligned}
$$

Consequently,

$$
\begin{aligned}
\frac{\partial}{\partial x} u(x, t)= & \int_{-\infty}^{\infty} \frac{-2(x-y)}{4 d(t-s) \sqrt{4 \pi d(t-s)}} e^{-\frac{(x-y)^{2}}{4 d(t-s)}} u(y, s) d y \\
& +\int_{s}^{t} \int_{-\infty}^{\infty} \frac{-2(x-y)}{4 d(t-r) \sqrt{4 \pi d(t-r)}} e^{-\frac{(x-y)^{2}}{4 d(t-r)}} F\left(u_{r}\right) d y d r .
\end{aligned}
$$

Then for $s \geq \tau$ and $t \in[s+1, s+5]$, we have

$$
\begin{aligned}
\left|\frac{\partial}{\partial x} u(x, t)\right| \leq & \int_{-\infty}^{\infty} \frac{2|y|}{4 d(t-s) \sqrt{4 \pi d(t-s)}} e^{-\frac{y^{2}}{4 d(t-s)}} d y \\
& +\int_{s}^{t} \int_{-\infty}^{\infty} \frac{2|y|}{4 d(t-r) \sqrt{4 \pi d(t-r)}} e^{-\frac{y^{2}}{4 d(t-r)}} d y d r \sup _{v \in C_{[0,1]}}\|F(v)\|_{X} \\
= & \frac{1}{\sqrt{\pi d(t-s)}}+\frac{2 \sqrt{t-s}}{\sqrt{\pi d}} \sup _{v \in C_{[0,1]}}\|F(v)\|_{X} \\
\leq & \frac{1}{\sqrt{\pi d}}+\frac{4}{\sqrt{\pi d}} \sup _{v \in C_{[0,1]}}\|F(v)\|_{X} \equiv M_{2} .
\end{aligned}
$$

Obviously, $s \geq \tau$ is arbitrary, which implies that $\left|\frac{\partial}{\partial x} u(x, t)\right| \leq M_{2}$ for any $x \in \mathbb{R}$ and any $t \geq \tau+1$. Moreover,

$$
\begin{aligned}
\frac{\partial}{\partial x} u(x, t)= & \int_{-\infty}^{\infty} \frac{-2(x-y)}{4 d(t-s) \sqrt{4 \pi d(t-s)}} e^{-\frac{(x-y)^{2}}{4 d(t-s)}} u(y, s) d y \\
& +\int_{s}^{t} \int_{-\infty}^{\infty} \frac{1}{\sqrt{4 \pi d(t-r)}} e^{-\frac{(x-y)^{2}}{4 d(t-r)}} \frac{\partial}{\partial y} F\left(u_{r}\right) d y d r .
\end{aligned}
$$

Hence,

$$
\begin{aligned}
\frac{\partial^{2}}{\partial x^{2}} u(x, t)= & \int_{-\infty}^{\infty} \frac{1}{\sqrt{4 \pi d(t-s)}}\left\{\frac{-2}{4 d(t-s)}+\frac{4(x-y)^{2}}{[4 d(t-s)]^{2}}\right\} e^{-\frac{(x-y)^{2}}{4 d(t-s)}} u(y, s) d y \\
& +\int_{s}^{t} \int_{-\infty}^{\infty} \frac{-2(x-y)}{4 d(t-r) \sqrt{4 \pi d(t-r)}} e^{-\frac{(x-y)^{2}}{4(t-r)}} \frac{\partial}{\partial y} F\left(u_{r}\right) d y d r .
\end{aligned}
$$

Thus, for $s \geq 2 \tau+1$ and $t \in[s+1, s+5]$,

$$
\begin{aligned}
\left|\frac{\partial^{2}}{\partial x^{2}} u(x, t)\right| \leq & \frac{1}{2 d(t-s)}+\int_{-\infty}^{\infty} \frac{4(x-y)^{2}}{[4 d(t-s)]^{2} \sqrt{4 \pi d(t-s)}} e^{-\frac{(x-y)^{2}}{4 d(t-s)}} d y \\
& +M_{2} M_{4} \int_{s}^{t} \int_{-\infty}^{\infty} \frac{2|y|}{4 d(t-r) \sqrt{4 \pi d(t-r)}} e^{-\frac{y^{2}}{4 d(t-r)}} d y d r \\
\leq & \frac{1}{d(t-s)}+M_{2} M_{4} \frac{2 \sqrt{t-s}}{\sqrt{\pi d}} \leq \frac{1}{d}+\frac{4 M_{2} M_{4}}{\sqrt{\pi d}} \equiv M_{3},
\end{aligned}
$$


where $M_{4}=\max \left\{\left|\partial_{1} g(u, v)\right|+\partial_{2} g(u, v) S^{\prime}(w): u, v \in[0,1], w \in[S(0), S(1)]\right\}$. By the arbitrariness of $s \geq 2 \tau+1$, we have $\left|\frac{\partial^{2}}{\partial x^{2}} u(x, t)\right| \leq M_{3}$ for any $x \in \mathbb{R}$ and any $t \geq 2(\tau+1)$. Since $u(x, t)$ satisfies

$$
\frac{\partial}{\partial t} u(x, t)=\frac{\partial^{2}}{\partial x^{2}} u(x, t)+g(u(x, t),(h * S(u))(x, t)), x \in \mathbb{R}, t>\tau,
$$

it follows that for any $x \in \mathbb{R}$ and any $t \geq 2(\tau+1)$,

$$
\left|\frac{\partial}{\partial t} u(x, t)\right| \leq M_{3}+\max _{u, v \in[0,1]}|g(u, S(v))| \equiv M_{1} .
$$

Constants $M_{1}, M_{2}$ and $M_{3}$ are independent of $x \in \mathbb{R}, t \geq 2(\tau+1)$ and $\varphi \in C_{[0,1]}$. Now we estimate $\frac{\partial^{2}}{\partial x \partial t} u(x, t)$ and $\frac{\partial^{3}}{\partial x^{3}} u(x, t)$. Notice that $\left|\frac{\partial}{\partial x} u(x, t)\right| \leq M_{2}$ for all $x \in \mathbb{R}$ and $t \geq \tau+1$. By (4.17), we have, for $t \geq 2 \tau+1$, that

$$
\begin{aligned}
\frac{\partial}{\partial x} u(x, t)= & \int_{-\infty}^{\infty} \frac{1}{\sqrt{4 \pi d(t-2 \tau-1)}} e^{-\frac{y^{2}}{4 d(t-2 \tau-1)}} \frac{\partial}{\partial x} u(x-y, 2 \tau+1) d y \\
& +\int_{2 \tau+1}^{t} \int_{-\infty}^{\infty} \frac{1}{\sqrt{4 \pi d(t-r)}} e^{-\frac{y^{2}}{4 d(t-r)}} \frac{\partial}{\partial x} F\left(u_{r}\right)(x-y) d y d r,
\end{aligned}
$$

which implies that $\frac{\partial}{\partial x} u(x, t)$ is a solution on $t \geq 2 \tau+1$ of the following equation:

$$
\begin{aligned}
\frac{\partial}{\partial t} v(x, t)=\quad & d \Delta v(x, t)+\partial_{1} g(u(x, t),(h * S(u))(x, t)) v(x, t) \\
& +\partial_{2} g(u(x, t),(h * S(u))(x, t))\left(h *\left(S^{\prime}(u) v\right)\right)(x, t)
\end{aligned}
$$

with initial value $v(x, 2 \tau+1+s)=\frac{\partial}{\partial x} u(x, 2 \tau+1+s), s \in[-\tau, 0]$. Applying a similar argument as in the previous part and combining the continuous second derivatives of $g(u, v)$ and $S(u)$, we can find a positive constant $M_{5}$, which is independent of $x, t$ and $\varphi \in C_{[0,1]}$, such that $\left|\frac{\partial^{2}}{\partial x \partial t} u(x, t)\right| \leq M_{5}$ and $\left|\frac{\partial^{3}}{\partial x^{3}} u(x, t)\right| \leq$ $M_{5}$ for any $x \in \mathbb{R}$ and $t \geq 3(\tau+1)$. Similarly, we can find a positive constant $M_{6}$, independent of $x, t$ and $\varphi \in C_{[0,1]}$, such that $\left|\frac{\partial^{2}}{\partial t^{2}} u(x, t)\right| \leq M_{6}$, $\left|\frac{\partial^{2}}{\partial t \partial x} u(x, t)\right| \leq M_{6}$ and $\left|\frac{\partial^{3}}{\partial x^{2} \partial t} u(x, t)\right| \leq M_{6}$ for any $x \in \mathbb{R}$ and $t \geq 3(\tau+1)$. Let $M=\max \left\{M_{1}, M_{2}, M_{3}, M_{5}, M_{6}\right\}$. The proof is complete.

Theorem 4.4. There exists an entire solution $\Phi(x, t)$ of (1.1) such that

$$
\underline{u}(x, t) \leq \Phi(x, t) \leq \bar{u}(x, t), \quad(x, t) \in \mathbb{R} \times(-\infty, T],
$$

where $\bar{u}(x, t)$ and $\underline{u}(x, t)$ are given in Lemma 4.1 and Lemma 4.2 , respectively. Moreover,

(i) $\frac{\partial}{\partial t} \Phi(x, t)>0$ on $\mathbb{R}^{2}$;

(ii) $\Phi(x, t)=\Phi(-x, t)$ on $\mathbb{R}^{2}$;

(iii) $\lim _{t \rightarrow \infty}\|\Phi(\cdot, t)-1\|_{L^{\infty}(\mathbb{R})}=0$ and for any $a>0$,

$$
\lim _{t \rightarrow-\infty}\|\Phi(\cdot, t)\|_{L^{\infty}([-a, a])}=0
$$


(iv) For each $a \in \mathbb{R}, \lim _{|x| \rightarrow \infty}\|\Phi(x, \cdot)-1\|_{L^{\infty}[a,+\infty)}=0$;

(v)

$$
\begin{aligned}
\lim _{t \rightarrow-\infty} & \left\{\sup _{x \geq 0}|\Phi(x, t)-\phi(x+c t+\omega)|\right. \\
& \left.+\sup _{x \leq 0}|\Phi(x, t)-\phi(-x+c t+\omega)|\right\}=0 .
\end{aligned}
$$

Proof. We denote a solution of (1.1) with initial data $\varphi \in C_{[0,1]}$ by $u(x, t ; \varphi)$. Define (4.18)

$u_{n}(x, t)=u\left(x, t ; \varphi_{n}\right), \quad \varphi_{n}(x, s)=\underline{u}(x, T-n+s), \quad(x, s) \in \mathbb{R} \times[-\tau, 0], n \in \mathbb{N}$.

Then $u_{n}(x, s)=\underline{u}(x, T-n+s)=\underline{u}(x, T-(n+1)+s+1) \leq u_{n+1}(x, 1+s)$, from which $u_{n}(x, n+s) \leq u_{n+1}(x, n+1+s)$ follows. On the other hand, we see $\underline{u}(x, T+s) \leq u_{n}(x, n+s) \leq \bar{u}(x, T+s)$. Thus, by Proposition 4.3 there exists a function $\varphi^{*} \in C_{[0,1]}$ to which $u_{n}(x, n+s)$ converges uniformly. Therefore, $\Phi(x, t):=u\left(x, t-T ; \varphi^{*}\right)$ is defined for all $t \geq T-\tau$.

To prove the continuation of a solution backward in time from $\varphi^{*}$, we show that given $T^{\prime}>0$, there is a function $\varphi^{T^{\prime}} \in C_{[0,1]}$ such that $\varphi^{*}(x, s)=u\left(x, T^{\prime}+s ; \varphi^{T^{\prime}}\right)$. Fix an integer $n_{1}>T^{\prime}+\tau$. For $n \geq n_{1}$, put

$$
w_{n}(x, s)=u_{n}\left(x, n-T^{\prime}+s\right)=u\left(x, n-T^{\prime}+s ; \varphi_{n}\right),
$$

where $\varphi_{n}$ is defined by (4.18). Then $u_{n}(x, n+s)=u\left(x, T^{\prime}+s ; w_{n}\right)$ and

$$
w_{n+1}(x, s)=u_{n+1}\left(x, n+1-T^{\prime}+s\right) \geq u_{n}\left(x, n-T^{\prime}+s\right)=w_{n}(x, s) .
$$

Thus, there is a $\varphi^{T^{\prime}} \in C_{[0,1]}$ such that $\lim _{n \rightarrow \infty}\left\|w_{n}-\varphi^{T^{\prime}}\right\|_{L^{\infty}(\mathbb{R} \times[-\tau, 0])}=0$. Here we note that it is easy to prove that for any $\varphi_{1}, \varphi_{2} \in C_{[0,1]}$,

$$
\left\|u\left(\cdot, t+\cdot ; \varphi_{1}\right)-u\left(\cdot, t+\cdot ; \varphi_{2}\right)\right\|_{L^{\infty}(\mathbb{R} \times[-\tau, 0])} \leq e^{\left(L_{1}+L_{2}\right) t}\left\|\varphi_{1}-\varphi_{2}\right\|_{L^{\infty}(\mathbb{R} \times[-\tau, 0])},
$$

where

$$
L_{1}=\max _{u \in[0,1], v \in[S(0), S(1)]}\left|\partial_{1} g(u, v)\right|
$$

and

$$
L_{2}=\max _{u \in[0,1]} S^{\prime}(u) \max _{u \in[0,1], v \in[S(0), S(1)]} \partial_{2} g(u, v) .
$$

It follows that $u_{n}(x, n+s)=u\left(x, T^{\prime}+s ; w_{n}\right), \lim _{n \rightarrow \infty}\left\|w_{n}-\varphi^{T^{\prime}}\right\|_{L^{\infty}(\mathbb{R} \times[-\tau, 0])}=$ 0 , and we see that $\varphi^{*}(x, s)=u\left(x, T^{\prime}+s ; \varphi^{T^{\prime}}\right)$. Hence, $\Phi(x, t)$ is defined for all $t \in \mathbb{R}$.

Now we show that $\frac{\partial}{\partial t} \Phi(x, t)>0$ on $\mathbb{R}^{2}$. Since $u(x, t)$ is a subsolution of (1.1), then $u_{n}(x, t)=u\left(x, t ; \varphi_{n}\right) \geq \underline{u}(x, T-n+t)$ for all $\mathbb{R} \times[-\tau,-T+n]$. Again since for any $\epsilon>0, \underline{u}(\cdot, \cdot+\epsilon) \geq \underline{u}(\cdot, \cdot)$ on $\mathbb{R}^{2}$, it follows that $u\left(x, \epsilon+s ; \varphi_{n}\right) \geq \varphi_{n}(x, s)$ for all $(x, s) \in \mathbb{R} \times[-\tau, 0]$. By comparison and the uniqueness of solutions, we have $u_{n}(x, t+\epsilon)=u\left(x, t ; u\left(\cdot, \epsilon+\cdot ; \varphi_{n}\right)\right) \geq u_{n}(x, t)$ for any $(x, t) \in \mathbb{R} \times(0,+\infty)$. It follows from the arbitrariness of $\epsilon$ that $u_{n}(x, t)$ is increasing in $t$. Consequently, it is easy to obtain $\frac{\partial}{\partial t} \Phi(x, t) \geq 0$ on $\mathbb{R}^{2}$. Since $\frac{\partial}{\partial t} \Phi(x, t)$ is a solution of the equation

$$
\begin{aligned}
\frac{\partial}{\partial t} v(x, t)= & d \Delta v(x, t)+\partial_{1} g(u(x, t),(h * S(u))(x, t)) v(x, t) \\
& +\partial_{2} g(u(x, t),(h * S(u))(x, t))\left(h * S^{\prime}(u) v\right)(x, t),
\end{aligned}
$$


combining $\partial_{2} g(u(x, t),(h * S(u))(x, t)) \geq 0$ and $S^{\prime}(u(x-y, t-r)) \geq 0$, then the strong maximum principle (Protter and Weinberger [33]) gives $\frac{\partial}{\partial t} \Phi(x, t)>0$ on $\mathbb{R}^{2}$. The proofs of (ii), (iii), (iv) and (v) are straightforward. This completes the proof.

Remark 4.5. In fact, the existence of the entire solution $\Phi$ can be proved as in Hamel and Nadirashvili 21] by applying Proposition 4.3 ,

\section{UNIQUENESS AND STABILITY OF ENTIRE SOLUTIONS}

In this section we show the uniqueness and stability of the entire solution $\Phi$ found in Theorem 4.4 under condition $\left(\mathrm{U}^{+}\right)$. In this section we always assume that $\widetilde{\Phi}(x, t)$ is an entire solution of (1.1) and satisfies $\left(\mathrm{U}^{+}\right)$and $0<\widetilde{\Phi}(x, t)<1$. We will use the method of Chen and Guo $[8$ to show that $\widetilde{\Phi}$ is only a translation of $\Phi$.

Lemma 5.1. Let $\beta_{0}$ be as in (1.9). Then there exists $T_{1} \in \mathbb{R}$ such that

$$
M(t)=\inf _{x \in \mathbb{R}, s \in[-\tau, 0]} \widetilde{\Phi}(x, t+s)<\beta_{0}, \quad \forall t \leq T_{1} .
$$

Proof. Let $\beta^{*}<\beta_{0}$ be a constant such that $g(u, S(u))>0$ in $u \in\left[\beta^{*}, 1\right)$. Denote by $\xi(\cdot)$ the solution of $\xi^{\prime}(t)=g\left(\xi(t), \int_{0}^{\tau} \int_{-\infty}^{\infty} h(y, s) S(\xi(t-s)) d y d s\right)$ with initial value $\xi(s)=\beta^{*}, s \in[-\tau, 0]$. Then by Smith [37, Corollary 2.2, p82], $\xi^{\prime}(\cdot) \geq 0$ in $(0, \infty)$, and hence, $\xi(\infty)=1$.

We argue by contradiction. Assuming that the assertion were not true, there would exist a sequence $\left\{t_{j}\right\}_{j=1}^{\infty}$ such that $\lim _{j \rightarrow \infty} t_{j}=-\infty$ and $M\left(t_{j}\right)>\beta^{*}$ for all $j$. By comparison, $M(t) \geq \inf _{s \in[-\tau, 0]} \xi\left(t-t_{j}+s\right)$ for all $t>t_{j}$. Fixing $t$ and letting $j \rightarrow \infty$ gives $M(t) \geq \lim _{j \rightarrow \infty} \inf _{s \in[-\tau, 0]} \xi\left(t-t_{j}+s\right)=\xi(\infty)=1$, which is a contradiction. This completes the proof.

From (1.8) and (5.1), the following functions are well-defined for all $t \leq T_{2}=$ $\min \left\{T_{0}, T_{1}\right\}$ :

$$
\begin{gathered}
\tilde{l}(t)=\min \left\{x: \widetilde{\Phi}(x, t+s)=\beta_{0}, s \in[-\tau, 0]\right\}, \\
\tilde{r}(t)=\max \left\{x: \widetilde{\Phi}(x, t+s)=\beta_{0}, s \in[-\tau, 0]\right\}, \\
q(t)=\frac{1}{2}[\tilde{r}(t)-\tilde{l}(t)], \quad m(t)=\frac{1}{2}[\tilde{r}(t)+\tilde{l}(t)] .
\end{gathered}
$$

Lemma 5.2. $\lim _{t \rightarrow-\infty} q(t)=\infty$.

Proof. Assuming that the assertion of the lemma were not true, then there exists an $L^{\prime}>0$ and a sequence $\left\{t_{j}\right\}_{j=1}^{\infty}$ such that $\lim _{j \rightarrow \infty} t_{j}=-\infty$ and $0<\tilde{r}\left(t_{j}\right)-\tilde{l}\left(t_{j}\right)<L^{\prime}$ for each integer $j>0$. Define

$$
\begin{aligned}
& \varphi\left(L^{\prime} ; y, s\right) \\
& \quad= \begin{cases}0 & \text { when }(y, s) \in\left[-L^{\prime}, L^{\prime}\right] \times[-\tau, 0] \\
\beta_{0} & \text { when }(y, s) \in\left(\left(-\infty,-\left(L^{\prime}+1\right)\right] \cup\left[L^{\prime}+1, \infty\right)\right) \times[-\tau, 0], \\
-\left(y+L^{\prime}\right) \beta_{0} & \text { when }(y, s) \in\left[-\left(L^{\prime}+1\right),-L^{\prime}\right] \times[-\tau, 0], \\
\left(y-L^{\prime}\right) \beta_{0} & \text { when }(y, s) \in\left[L^{\prime}, L^{\prime}+1\right] \times[-\tau, 0]\end{cases}
\end{aligned}
$$


Denote by $u(x, t ; \varphi)$ the solution of (1.1) with initial $\varphi$. Since $c>0$, by the following Lemma 5.3, there exists a constant $T\left(L^{\prime}\right)$ such that $\beta_{0} \leq u(x, t ; \varphi) \leq$ 1 for any $t \geq T\left(L^{\prime}\right)$. Comparing $\varphi\left(L^{\prime} ; \cdot, s\right)$ with $\widetilde{\Phi}\left(m\left(t_{j}\right)+\cdot, t_{j}+s\right)$, we have $\varphi\left(L^{\prime} ; \cdot, s\right) \leq \widetilde{\Phi}\left(m\left(t_{j}\right)+\cdot, t_{j}+s\right)$ for $s \in[-\tau, 0]$. Thus, by comparison, we have $\widetilde{\Phi}\left(m\left(t_{j}\right)+\cdot, t_{j}+t\right) \geq u(\cdot, t ; \varphi)$ for all $t>0$. This implies that $M\left(t_{j}+T\left(L^{\prime}\right)\right) \geq \beta_{0}$ for all integer $j \geq 0$, which contradicts (5.1). The proof is complete.

Let

$$
\begin{aligned}
& \alpha_{1}=\min \{u \mid g(u, S(u))=0, u \in(0,1)\}, \\
& \beta_{1}=\max \{u \mid g(u, S(u))=0, u \in(0,1)\}
\end{aligned}
$$

and

$$
\begin{aligned}
C_{[0,1]}^{a}=\left\{\varphi \in C_{[0,1]} \mid \varphi \leq \frac{\alpha_{0}+\alpha_{1}}{2} \text { in }(-\infty,-a] \times[-\tau, 0],\right. & \\
& \left.\varphi \geq \beta_{0} \text { in }[0, \infty) \times[-\tau, 0]\right\} .
\end{aligned}
$$

Obviously, $\alpha_{0}<\alpha_{1} \leq \beta_{1}<\beta_{0}$. The following lemma was proved by Wang et al. [42]; see also Chen [7, Theorem 3.1] and Smith and Zhao [38, Theorem 3.3]. We note that though Theorem 3.1 in Chen [7] and Theorem 3.3 in Smith and Zhao [38] require that the quasimonotone condition holds on a larger domain than $[0,1] \times$ $[S(0), S(1)]$ and $[0,1]^{2}$, respectively (see [7, (D3), p. 152] and [38, (H1), p. 515]), their results still hold under (F1) if supersolutions and subsolutions are chosen as in Wang et al. 42. That is, take the smaller between 1 and the supersolution in Chen [7] and Smith and Zhao [38] (or the larger between 0 and the subsolution) as a new supersolution (a new subsolution).

Lemma 5.3. There exists a positive constant $\nu$ such that for any $\varphi \in C_{[0,1]}$ with

$$
\liminf _{x \rightarrow \infty} \min _{s \in[-\tau, 0]} \varphi(x, s)>\beta_{1} \quad \text { and } \quad \limsup _{x \rightarrow-\infty} \max _{s \in[-\tau, 0]} \varphi(x, s)<\alpha_{1},
$$

the solution of (1.1) with initial value $\varphi$ satisfies

$$
|u(x, t ; \varphi)-\phi(x+c t+\xi)| \leq K e^{\nu t} \quad \forall x \in \mathbb{R}, t \geq 0,
$$

for some $K=K(\varphi)>0$ and some $\xi=\xi(\varphi) \in \mathbb{R}$.

Now we consider $\varphi \in C_{[0,1]}^{a}$. Carefully observe the proof of Theorem 3.1 in [7, the proof of Theorem 3.3 in [38] and the proof of Theorem 4.5 in [42]. We can find two constants $K^{\prime}>0$ and $\xi_{0}>0$ such that for all $\varphi \in C_{[0,1]}^{a}, K(\varphi) \leq K^{\prime}$ and $|\xi(\varphi)| \leq \xi_{0}$. Let $K_{0}=\max \left\{\xi_{0}, K^{\prime}\right\}$.

Lemma 5.4. There exist positive constants $\nu$ and $K_{0}$ such that for any $\varphi \in C_{[0,1]}^{a}$, the solution of (1.1) with initial value $\varphi$ satisfies

$$
|u(x, t ; \varphi)-\phi(x+c t+\xi)| \leq K_{0} e^{\nu t} \quad \forall x \in \mathbb{R}, t \geq 0
$$

for some $\xi=\xi(\varphi) \in \mathbb{R}$ satisfying $|\xi| \leq K_{0}$. 
From (1.8), $l(t) \leq \tilde{l}(t)<\tilde{r}(t) \leq r(t)$. Hence, for all $t \ll-1$,

$$
\begin{array}{ll}
\widetilde{\Phi}(m(t)+x, t+s) \geq \beta_{0} & \text { if }|x| \geq q(t), \\
\widetilde{\Phi}(m(t)+x, t+s) \leq \alpha_{0} & \text { if }|x| \leq q(t)-a .
\end{array}
$$

Since $q(t) \rightarrow \infty$ as $t \rightarrow-\infty$, there exists $T_{3} \leq T_{2}$ such that for any $t \leq T_{3}$, $m(t) \in[\min \{l(t)+a, r(t)-a\}, \max \{l(t)+a, r(t)-a\}]$.

Assume $r \leq T_{3}$. Define

$$
\psi(x, s)= \begin{cases}\widetilde{\Phi}(x+m(r), r+s) & \text { if } x \geq-q(r)+a, \\ \widetilde{\Phi}(-q(r)+a+m(r), r+s) & \\ -\frac{\eta \pi_{2}(0)}{6(M+1)}\left[\cos \left(\sqrt{\frac{6(M+1)}{\eta}}(x+q(r)-a)\right)-1\right] & \text { if } x<-q(r)+a, \\ +\frac{\eta \pi_{1}(s)}{6(M+1)} \sin \left(\frac{6(M+1)}{\eta}(x+q(r)-a)\right) & \end{cases}
$$

where $s \in[-\tau, 0], \eta=\min \left\{\alpha_{1}-\alpha_{0}, \min _{s \in[-\tau, 0]} \widetilde{\Phi}(-q(r)+a+m(r), r+s)\right\}, M$ is defined in Proposition 4.3, and

$$
\pi_{1}(s)=\left.\frac{\partial}{\partial x} \widetilde{\Phi}(x+m(r), r+s)\right|_{x=-q(r)+a}, \pi_{2}(0)=\left.\frac{\partial^{2}}{\partial x^{2}} \widetilde{\Phi}(x+m(r), r)\right|_{x=-q(r)+a} .
$$

Lemma 5.5. Assume that $h(x, t)=J(x) \delta(t-\tau)$; then for every $\varepsilon$ and $H>0$, there exists $r_{0}(\varepsilon, H)<0$ such that for any $r \leq r_{0}$,

$$
|u(x, t ; \psi)-\widetilde{\Phi}(x+m(r), r+t)| \leq \varepsilon \quad \forall t \in[0, H], x \in[0, \infty) .
$$

Proof. Now let $u(x, t ; \psi)$ be the solution of (1.1) with initial value $\psi(x, s)$. By Pazy [30, Theorem 3.1], $v(t)=\int_{0}^{t} T(t-s) F\left(u_{s}\right) d s$ is Hölder continuous on $[0,+\infty)$. Also, since $\psi(x, 0) \in D\left(d \Delta_{X}\right)$, then $T(t) \psi(0)$ is Lipschitz continuous on $[0,+\infty)$. Thus, $u(x, t ; \psi)$ is Hölder continuous on $[0,+\infty)$. In view of the Lipschitz continuity of $\psi$ on $[-\tau, 0]$, it follows that $f(t)=F\left(u_{t}\right)$ is Hölder continuous on $[0,+\infty)$. Following Pazy [30, Corollary 3.3], $u(x, t ; \psi)$ is a classical solution of (1.1) in $(0,+\infty)$.

Let $v(x, t)=u(x, t ; \psi)-\widetilde{\Phi}(x+m(r), r+t)$. Then $v(x, s)=0$ for all $(x, s) \in$ $(-q(r)+a,+\infty) \times[-\tau, 0]$ and

$$
\begin{aligned}
\frac{\partial}{\partial t} v(x, t)-d \Delta v(x, t)= & g(u(x, t),(h * S(u))(x, t)) \\
& -g(\widetilde{\Phi}(x+m(r), r+t),(h * S(\widetilde{\Phi}))(x+m(r), r+t)) .
\end{aligned}
$$

Take $v^{-}(x, t)=\max \{-v(x, t), 0\}$ and $v^{+}(x, t)=\max \{v(x, t), 0\}$. Then

$$
\frac{\partial}{\partial t} v(x, t)-d \Delta v(x, t) \leq L_{1} v(x, t)+L_{2} \int_{-\infty}^{\infty} J(y) v^{+}(x-y, t-\tau) d y
$$

if $v(x, t)>0$ and

$$
\frac{\partial}{\partial t} v(x, t)-d \Delta v \geq L_{1} v(x, t)-L_{2} \int_{-\infty}^{\infty} J(y) v^{-}(x-y, t-\tau) d y
$$

if $v(x, t)<0$, where $L_{1}$ and $L_{2}$ are defined in Theorem 4.4 
Given $\varepsilon>0$ and $H>0$, there exists $m \in \mathbb{N}$ such that $m-1=\left[\frac{H}{\tau}\right]$. Define $v_{j}^{+}(x, t)=v^{+}(x, t+(j-1) \tau)$ for any $(x, t) \in \mathbb{R} \times[-\tau, \infty)$ and $j \in\{1, \cdots, m\}$. Let $w\left(x, t ; v_{j}^{+}(x, 0)\right)$ be a solution of the following linear equation:

$$
\frac{\partial}{\partial t} w(x, t)-d \Delta w(x, t)=L_{1} w(x, t)+L_{2} \int_{-\infty}^{\infty} J(y) v_{j}^{+}(x-y, t-\tau) d y, \quad t \geq 0,
$$

with initial value $v_{j}^{+}(x, 0)$. It is easy to see that $v_{j}^{+}(x, t)$ is a subsolution of (5.3) on $(x, t) \in \mathbb{R} \times[0, \infty)$. Then for $(x, t) \in \mathbb{R} \times[0, \tau]$,

$$
\begin{aligned}
& v_{j}^{+}(x, t) \leq w\left(x, t ; v_{j}^{+}(x, 0)\right)=e^{L_{1} t} \int_{-\infty}^{\infty} \frac{1}{\sqrt{4 d \pi t}} e^{-\frac{(x-y)^{2}}{4 d t}} v_{j}^{+}(y, 0) d y \\
& +\int_{0}^{t} e^{L_{1}(t-s)} \int_{-\infty}^{\infty} \frac{1}{\sqrt{4 d \pi(t-s)}} \\
& \times e^{-\frac{(x-y)^{2}}{4 d(t-s)}} L_{2} \int_{-\infty}^{\infty} J(z) v_{j}^{+}(y-z, s-\tau) d z d y d s \\
& \leq e^{L_{1} \tau} \int_{-\infty}^{\infty} \frac{1}{\sqrt{4 d \pi t}} e^{-\frac{(x-y)^{2}}{4 d t}} v_{j}^{+}(y, 0) d y \\
& +L_{2} e^{L_{1} \tau} \int_{0}^{t} \int_{-\infty}^{\infty} \frac{1}{\sqrt{4 d \pi(t-s)}} \\
& \times e^{-\frac{(x-y)^{2}}{4 d(t-s)}} \int_{-\infty}^{\infty} J(z) v_{j}^{+}(y-z, s-\tau) d z d y d s .
\end{aligned}
$$

Claim. For given $\varepsilon_{j}>0$ and $Y_{j} \leq 0$, there exists $Y_{j-1}$ with $Y_{j-1}<Y_{j}$ which is only dependent on $\varepsilon_{j}$ and $Y_{j}$, such that if

$$
v_{j-1}^{+}(x, t) \leq \varepsilon_{j-1}:=\min \left\{\varepsilon_{j} /\left(5 e^{L_{1} \tau}\right), \varepsilon_{j} /\left(5 L_{2} \tau e^{L_{1} \tau}\right)\right\}
$$

for any $(x, t) \in\left[Y_{j-1},+\infty\right) \times[0, \tau]$, there are $v_{j}^{+}(x, t) \leq \varepsilon_{j}$ for any $(x, t) \in$ $\left[Y_{j},+\infty\right) \times[0, \tau]$.

We now prove the Claim. Take $Z_{j}>0$ such that

$$
L_{2} \tau e^{L_{1} \tau} \int_{-\infty}^{-Z_{j}}+\int_{Z_{j}}^{+\infty} J(y) d y \leq \varepsilon_{j} / 5
$$

Furthermore, take $\Lambda_{1 j}>0$ and $\Lambda_{2 j}>0$ such that $\int_{\frac{\Lambda_{1 j}}{\sqrt{4 d \tau}}}^{+\infty} e^{-y^{2}} d y \leq \frac{\sqrt{\pi} \varepsilon_{j}}{5 e^{L_{1} \tau}}$ and $\int_{\frac{\Lambda_{2 j}}{\sqrt{4 d \tau}}}^{\infty} e^{-y^{2}} d y \leq \frac{\sqrt{\pi} \varepsilon_{j}}{5 L_{2} \tau e^{L_{1} \tau}}$. Let $Y_{j-1}=\min \left\{Y_{j}-\Lambda_{1 j}, Y_{j}-Z_{j}-\Lambda_{2 j}\right\}$. Notice that $v_{j}^{+}(x, t-\tau)=v_{j-1}^{+}(x, t)$. If $v_{j-1}^{+}(x, t) \leq \varepsilon_{j-1}$ for any $(x, t) \in\left[Y_{j-1},+\infty\right) \times[0, \tau]$, 
then for any $(x, t) \in\left[Y_{j},+\infty\right) \times[0, \tau]$, we have

$$
\begin{gathered}
e^{L_{1} \tau} \int_{Y_{j-1}}^{\infty} \frac{1}{\sqrt{4 d \pi t}} e^{-\frac{(x-y)^{2}}{4 d t}} v_{j}^{+}(y, 0) d y \leq \varepsilon_{j} / 5 \\
e^{L_{1} \tau} \int_{-\infty}^{Y_{j-1}} \frac{1}{\sqrt{4 d \pi t}} e^{-\frac{(x-y)^{2}}{4 d t}} v_{j}^{+}(y, 0) d y \leq e^{L_{1} \tau} \int_{x-Y_{j-1}}^{+\infty} \frac{1}{\sqrt{4 d \pi t}} e^{-\frac{y^{2}}{4 d t}} d y \\
\leq e^{L_{1} \tau} \int_{Y_{j}-Y_{j-1}}^{+\infty} \frac{1}{\sqrt{4 d \pi t}} e^{-\frac{y^{2}}{4 d t}} d y=\frac{e^{L_{1} \tau}}{\sqrt{\pi}} \int_{\frac{Y_{j}-Y_{j-1}}{\sqrt{4 d t}}}^{+\infty} e^{-y^{2}} d y \\
=\frac{e^{L_{1} \tau}}{\sqrt{\pi}} \int_{\frac{Y_{j}-Y_{j-1}}{\sqrt{4 d \tau}}}^{+\infty} e^{-y^{2}} d y \leq \frac{e^{L_{1} \tau}}{\sqrt{\pi}} \int_{\frac{\Lambda_{1 j}}{\sqrt{4 d \tau}}}^{+\infty} e^{-y^{2}} d y \leq \varepsilon_{j} / 5, \\
L_{2} e^{L_{1} \tau} \int_{0}^{t} \int_{-\infty}^{\infty} \frac{1}{\sqrt{4 d \pi(t-s)}} e^{-\frac{(x-y)^{2}}{4 d(t-s)}} \\
\times\left[\int_{-\infty}^{Z_{j}}+\int_{Z_{j}}^{\infty} J(z) v_{j}^{+}(y-z, s-\tau) d z\right] d y d s \\
\leq L_{2} \tau e^{L_{1} \tau} \int_{-\infty}^{Z_{j}}+\int_{Z_{j}}^{\infty} J(z) d z \leq \varepsilon_{j} / 5, \\
L_{2} e^{L_{1} \tau} \int_{0}^{t} \int_{Y_{j-1}+Z_{j}}^{\infty} \frac{1}{\sqrt{4 d \pi(t-s)}} e^{-\frac{(x-y)^{2}}{4 d(t-s)}} \\
\times \int_{-Z_{j}}^{Z_{j}} J(z) v_{j}^{+}(y-z, s-\tau) d z d y d s \leq \varepsilon_{j} / 5
\end{gathered}
$$

and

$$
\begin{aligned}
& L_{2} e^{L_{1} \tau} \int_{0}^{t} \int_{-\infty}^{Y_{j-1}+Z_{j}} \frac{1}{\sqrt{4 d \pi(t-s)}} e^{-\frac{(x-y)^{2}}{4 d(t-s)}} \int_{-Z_{j}}^{Z_{j}} J(z) v_{j}^{+}(y-z, s-\tau) d z d y d s \\
& \quad \leq L_{2} e^{L_{1} \tau} \int_{0}^{t} \int_{x-\left(Y_{j-1}+Z_{j}\right)}^{\infty} \frac{1}{\sqrt{4 d \pi(t-s)}} e^{-\frac{y^{2}}{4 d(t-s)}} d y d s \\
& \leq \frac{L_{2} \tau e^{L_{1} \tau}}{\sqrt{\pi}} \int_{\frac{x-\left(Y_{j-1}+Z_{j}\right)}{\sqrt{4 d \tau}}}^{\infty} e^{-y^{2}} d y \leq \frac{L_{2} \tau e^{L_{1} \tau}}{\sqrt{\pi}} \int_{\frac{\Lambda_{2 j}}{\sqrt{4 d \tau}}}^{\infty} e^{-y^{2}} d y \leq \varepsilon_{j} / 5,
\end{aligned}
$$

which implies that for any $(x, t) \in\left[Y_{j},+\infty\right) \times[0, \tau], v_{j}^{+}(x, t) \leq \varepsilon_{j}$. Obviously, $\varepsilon_{j-1}$ and $Y_{j-1}$ are only dependent on $\varepsilon_{j}$ and $Y_{j}$. Thus, we have shown the Claim.

Let $\varepsilon_{m}=\varepsilon$ and $Y_{m}=0$. According to the Claim, we can choose $\left\{\left(\varepsilon_{j}, Y_{j}\right): j=1\right.$, $\cdots, m\}$ such that when $v_{j-1}^{+}(x, t) \leq \varepsilon_{j-1}<\varepsilon_{j}$ for any $(x, t) \in\left[Y_{j-1},+\infty\right) \times[0, \tau]$, there are $v_{j}^{+}(x, t) \leq \varepsilon_{j}$ for any $(x, t) \in\left[Y_{j},+\infty\right) \times[0, \tau]$. In view of $Y_{j-1}<Y_{j}, \varepsilon_{j} \leq \varepsilon$ and $v_{j}^{+}(x, t)=v_{1}^{+}(x, t+(j-1) \tau)=v^{+}(x, t+(j-1) \tau)$, then if $v_{1}^{+}(x, t) \leq \varepsilon_{1} \leq \varepsilon$ for any $(x, t) \in\left[Y_{1},+\infty\right) \times[0, \tau]$, there is $v^{+}(x, t) \leq \varepsilon$ for any $(x, t) \in[0,+\infty) \times$ $[0, m \tau]$. By virtue of $H \in[(m-1) \tau, m \tau]$, for any $(x, t) \in[0,+\infty) \times[0, H]$, there is $v^{+}(x, t) \leq \varepsilon$ if $v_{1}^{+}(x, t) \leq \varepsilon_{1} \leq \varepsilon$ for any $(x, t) \in\left[Y_{1},+\infty\right) \times[0, \tau]$. To complete the proof of the lemma, we only need to show that there exists $\bar{r}_{1}(\varepsilon, H)<0$ such that 
for any $r \leq \bar{r}_{1}, v_{1}^{+}(x, t)=v^{+}(x, t) \leq \varepsilon_{1}$ for any $(x, t) \in\left[Y_{1},+\infty\right) \times[0, \tau]$. Define $v_{0}^{+}(x, t)=v^{+}(x, t-\tau)$ for $(x, t) \in \mathbb{R} \times[0, \tau]$. Then by a similar argument, we can choose $\varepsilon_{0}<\varepsilon_{1}$ and $Y_{0}<Y_{1}$ such that when $v_{0}^{+}(x, t) \leq \varepsilon_{0}$ for $(x, t) \in\left[Y_{0}, \infty\right) \times[0, \tau]$, there are $v_{1}^{+}(x, t)=v^{+}(x, t) \leq \varepsilon_{1}$ for any $(x, t) \in\left[Y_{1},+\infty\right) \times[0, \tau]$. Note that $\varepsilon_{0}$ and $Y_{0}$ are only dependent on $\varepsilon$ and $H$. It is sufficient to set $\bar{r}_{1}(\varepsilon, H)<T_{3}$ such that for any $r \leq \bar{r}_{1},-q(r)+a \leq Y_{0}$.

Similarly, we can choose $\bar{r}_{2}(\varepsilon, H)<T_{3}$ such that for any $r \leq \bar{r}_{2}$, there is $v^{-}(x, t) \leq \varepsilon$ for any $x \in[0, \infty)$ and $t \in[0, H]$. Let $r_{0}(\varepsilon, H)=\min \left\{\bar{r}_{1}, \bar{r}_{2}\right\}$. This completes the proof.

Lemma 5.6. Assume that $h(x, t)=J(x) \delta(t-\tau)$; then

$$
\lim _{t \rightarrow-\infty} \inf _{z \in \mathbb{R}, r \in \mathbb{R}}\|\widetilde{\Phi}(z+\cdot, t+\cdot)-\Phi(\cdot, r+\cdot)\|_{L^{\infty}(\mathbb{R} \times[-\tau, 0])}=0 .
$$

Proof. Let $\varepsilon>0$ be arbitrarily small. Set $H$ such that $K_{0} e^{-\nu H} e^{\nu \tau}=\varepsilon$. Choose $r_{1} \ll-1$ such that $\phi\left(-q(r)+c H+2 K_{0}\right)<\varepsilon$ for $r \leq r_{1}$. Fix any $r \leq \min \left\{r_{0}, r_{1}\right\}$. By Lemma 5.5 .

$$
|u(x, H+s ; \psi)-\widetilde{\Phi}(x+m(r), r+H+s)| \leq \varepsilon \quad \forall x \geq 0, s \in[-\tau, 0] .
$$

On the other hand, for some $\xi \in\left[-K_{0}, K_{0}\right]$,

$$
\begin{aligned}
|u(x, H+s ; \psi)-\phi(x-q(r)+c H+c s-\xi)| \leq K_{0} e^{-\nu(H+s)} & \leq \varepsilon \\
\forall x & \in \mathbb{R}, s \in[-\tau, 0],
\end{aligned}
$$

since $\psi(x, s) \leq \frac{\alpha_{0}+\alpha_{1}}{2}$ for all $x \leq q(r)-a$ and $s \in[-\tau, 0], \psi(x, s) \geq \beta_{0}$ for all $x \geq q(r)$ and $s \in[-\tau, 0]$. Thus, for all $x \geq 0$ and $s \in[-\tau, 0]$,

$$
|\widetilde{\Phi}(x+m(r), r+H+s)-\phi(x-q(r)+c H+c s-\xi)| \leq 2 \varepsilon .
$$

Similarly, for all $x \leq 0$ and $s \in[-\tau, 0]$, there exists some $\eta \in\left[-K_{0}, K_{0}\right]$ such that

$$
|\widetilde{\Phi}(x+m(r), r+H+s)-\phi(-x-q(r)+c H+c s-\eta)| \leq 2 \varepsilon .
$$

Since

$$
\begin{aligned}
& \phi(x-q(r)+c H+c s-\xi)=\phi((x-(\xi-\eta) / 2)-q(r)+c H+c s-(\xi+\eta) / 2), \\
& \phi(-x-q(r)+c H+c s-\eta)=\phi(-(x-(\xi-\eta) / 2)-q(r)+c H+c s-(\xi+\eta) / 2),
\end{aligned}
$$

then for $x \geq-(\xi-\eta) / 2$ and $s \in[-\tau, 0]$,

$|\widetilde{\Phi}(x+(\xi-\eta) / 2+m(r), r+H+s)-\phi(x-q(r)+c H+c s-(\xi+\eta) / 2)| \leq 2 \varepsilon$,

and for $x \leq-(\xi-\eta) / 2$ and $s \in[-\tau, 0]$,

$$
|\widetilde{\Phi}(x+(\xi-\eta) / 2+m(r), r+H+s)-\phi(-x-q(r)+c H+c s-(\xi+\eta) / 2)| \leq 2 \varepsilon .
$$

If $-(\xi-\eta) / 2 \geq 0$, then for $x \in[0,-(\xi-\eta) / 2] \subset\left[0, K_{0}\right]$ and $s \in[-\tau, 0]$,

$$
|\phi(x-q(r)+c H+c s-(\xi+\eta) / 2)-\phi(-x-q(r)+c H+c s-(\xi+\eta) / 2)| \leq \varepsilon .
$$


Thus, for $x \geq 0$ and $s \in[-\tau, 0]$,

$|\widetilde{\Phi}(x+(\xi-\eta) / 2+m(r), r+H+s)-\phi(x-q(r)+c H+c s-(\xi+\eta) / 2)| \leq 3 \varepsilon$

and

$$
\begin{aligned}
& \mid \widetilde{\Phi}(x+(\xi-\eta) / 2+m(r), r+H+s) \\
& -[\phi(x-q(r)+c H+c s-(\xi+\eta) / 2)+\phi(-x-q(r) \\
& +c H+c s-(\xi+\eta) / 2)] \mid \leq 4 \varepsilon .
\end{aligned}
$$

Obviously, for $x \leq 0$ and $s \in[-\tau, 0]$,

$|\widetilde{\Phi}(x+(\xi-\eta) / 2+m(r), r+H+s)-\phi(-x-q(r)+c H+c s-(\xi+\eta) / 2)| \leq 3 \varepsilon$

and

$$
\begin{aligned}
& \mid \widetilde{\Phi}(x+(\xi-\eta) / 2+m(r), r+H+s) \\
& -[\phi(x-q(r)+c H+c s-(\xi+\eta) / 2)+\phi(-x-q(r) \\
& +c H+c s-(\xi+\eta) / 2)] \mid \leq 4 \varepsilon .
\end{aligned}
$$

For the case $-(\xi-\eta) / 2<0$, we can obtain the same estimates. Define $\theta=\theta(r, s)$ with $p(\theta+s)=-q(r)+c H+c s-(\xi+\eta) / 2$, where $p$ is defined in Section 4 . It is obvious that $\theta \rightarrow-\infty$ is uniform for $s \in[-\tau, 0]$ as $r \rightarrow-\infty$. Again since $0<p(t)-c t-\omega \rightarrow 0$ as $t \rightarrow-\infty$, there exists $r_{2}<\min \left\{r_{0}, r_{1}\right\}$ such that for $r \leq r_{2}$ and $s \in[-\tau, 0], \theta<T$ and

$$
\begin{aligned}
& |\phi( \pm x-q(r)+c H+c s-(\xi+\eta) / 2)-\phi( \pm x+c \theta+c s+\omega)| \\
= & |\phi( \pm x+p(\theta+s))-\phi( \pm x+c \theta+c s+\omega)| \leq \varepsilon,
\end{aligned}
$$

which implies that

$$
\begin{aligned}
& \mid \widetilde{\Phi}(x+(\xi-\eta) / 2+m(r), r+H+s) \\
& \quad-\max \{\phi(x+c \theta+c s+\omega), \phi(-x+c \theta+c s+\omega)\} \mid \leq 4 \varepsilon
\end{aligned}
$$

and

$$
\begin{aligned}
\mid \widetilde{\Phi}(x+(\xi-\eta) / 2+m(r), r+H+s) & \\
& -[\phi(x+p(\theta+s))+\phi(-x+p(\theta+s))] \mid \leq 5 \varepsilon .
\end{aligned}
$$

Therefore, for any $r \leq r_{2}$ and $s \in[-\tau, 0]$,

$$
|\widetilde{\Phi}(x+(\xi-\eta) / 2+m(r), r+H+s)-\Phi(x, \theta+s)| \leq 5 \varepsilon .
$$

Consequently, for any $t \leq r_{2}+H$, we have

$$
\inf _{z \in \mathbb{R}, \theta \leq 0}\|\widetilde{\Phi}(z+\cdot, t+\cdot)-\Phi(\cdot, \theta+\cdot)\|_{L^{\infty}(\mathbb{R} \times[-\tau, 0])} \leq 5 \varepsilon .
$$

Hence,

$$
\sup _{t \leq r_{2}+H} \inf _{z \in \mathbb{R}, \theta \leq 0}\|\widetilde{\Phi}(z+\cdot, t+\cdot)-\Phi(\cdot, \theta+\cdot)\|_{L^{\infty}(\mathbb{R} \times[-\tau, 0])} \leq 5 \varepsilon
$$

which implies that the assertion of the lemma holds. The proof is complete. 
Lemma 5.7. There exist constants $\delta_{0}>0, \varrho_{0}>0$ and $\sigma_{0}>0$ such that for any $r \in \mathbb{R}, \delta \in\left(0, \delta_{0}\right]$ and $\sigma \geq \sigma_{0}$,

$$
\begin{aligned}
& W^{+}(x, t)=\min \left\{\Phi\left(x, r+t+\sigma \delta\left[1-e^{-\varrho_{0} t}\right]\right)+\delta e^{-\varrho_{0} t}, 1\right\}, \\
& W^{-}(x, t)=\max \left\{\Phi\left(x, r+t-\sigma \delta\left[1-e^{-\varrho_{0} t}\right]\right)-\delta e^{-\varrho_{0} t}, 0\right\}
\end{aligned}
$$

are a pair of supersolutions and subsolutions of (1.1) on $\mathbb{R} \times[0,+\infty)$.

Proof. We only prove that $W^{+}(x, t)$ is a supersolution of (1.1) on $\mathbb{R} \times(0,+\infty)$, since a similar argument can be used for $W^{-}(x, t)$.

Since

$$
\begin{aligned}
& \lim _{(u, v, r, s, \varpi, \varrho) \rightarrow\left(0+, S(0), 0+, S(0), S^{\prime}(0), 0\right)}\left[\partial_{1} g(u, v)+\varpi e^{\varrho \tau} \partial_{2} g(r, s)+\varrho\right] \\
& \quad=\partial_{1} g(0, S(0))+S^{\prime}(0) \partial_{2} g(0, S(0))<0
\end{aligned}
$$

and

$$
\begin{aligned}
& \lim _{(u, v, r, s, \varpi, \varrho) \rightarrow\left(1-, S(1), 1-, S(1), S^{\prime}(1), 0\right)}\left[\partial_{1} g(u, v)+\varpi e^{\varrho \tau} \partial_{2} g(r, s)+\varrho\right] \\
& \quad=\partial_{1} g(1, S(1))+S^{\prime}(1) \partial_{2} g(1, S(1))<0,
\end{aligned}
$$

we can fix $\varrho_{0}>0$ and $\delta_{1}>0$ such that

$$
\partial_{1} g(u, v)+\varpi e^{\varrho_{0} \tau} \partial_{2} g(r, s)<-\varrho_{0}
$$

for any

$$
\begin{aligned}
(u, v, r, s, \varpi) \in & {\left[0, \delta_{1}\right] \times\left[S(0), S(0)+\delta_{1}\right] \times\left[0, \delta_{1}\right] } \\
& \times\left[S(0), S(0)+\delta_{1}\right] \times\left[S^{\prime}(0)-\delta_{1}, S^{\prime}(0)+\delta_{1}\right]
\end{aligned}
$$

and

$$
\begin{aligned}
(u, v, r, s, \varpi) \in & {\left[1-\delta_{1}, 1\right] \times\left[S(1)-\delta_{1}, S(1)\right] \times\left[1-\delta_{1}, 1\right] } \\
& \times\left[S(1)-\delta_{1}, S(1)\right] \times\left[S^{\prime}(1)-\delta_{1}, S^{\prime}(1)+\delta_{1}\right] .
\end{aligned}
$$

Let $\delta_{0} \in\left(0, \delta_{1}\right)$ satisfy

$$
\delta_{0} e^{\varrho_{0} \tau}\left[1+\max _{u \in[0,1]}\left|S^{\prime}(u)\right|+\max _{u \in[0,1]}\left|S^{\prime \prime}(u)\right|\right] \leq \delta_{1} / 4 .
$$

Since $\lim _{t \rightarrow \infty}\|\Phi(\cdot, t)-1\|_{L^{\infty}(\mathbb{R})}=0, \lim _{t \rightarrow \infty}\|(h * S(\Phi))(\cdot, t)-S(1)\|_{L^{\infty}(\mathbb{R})}=0$ and $\lim _{t \rightarrow \infty}\left\|\left(h * S^{\prime}(\Phi)\right)(\cdot, t)-S^{\prime}(1)\right\|_{L^{\infty}(\mathbb{R})}=0$, there exists $T_{4}>0$ such that for $(x, t) \in \mathbb{R} \times\left(T_{4}, \infty\right)$,

$$
\begin{gathered}
\Phi(x, t) \in\left[1-\delta_{1}, 1\right],(h * S(\Phi))(x, t) \in\left[S(1)-\delta_{1}, S(1)\right], \\
\left(h * S^{\prime}(\Phi)\right)(x, t) \in\left[S^{\prime}(1)-\delta_{1} / 2, S^{\prime}(1)+\delta_{1} / 2\right] .
\end{gathered}
$$

Since $\lim _{\xi \rightarrow-\infty}(h * \phi)(\xi)=0, \quad \lim _{\xi \rightarrow-\infty}(h * S(\phi))(\xi)=S(0)$, $\lim _{\xi \rightarrow-\infty}\left(h * S^{\prime}(\phi)\right)(\xi)=S^{\prime}(0), \quad \lim _{\xi \rightarrow+\infty}(h * S(\phi))(\xi)=S(1)$ and $\lim _{\xi \rightarrow+\infty}\left(h * S^{\prime}(\phi)\right)(\xi)=S^{\prime}(1)$, there exists $X_{1}>0$ such that for $\xi \geq X_{1}$,

$$
\begin{gathered}
\phi(\xi) \in\left[1-\delta_{1}, 1\right], \quad(h * S(\phi))(\xi) \in\left[S(1)-\delta_{1}, S(1)\right], \\
\left(h * S^{\prime}(\phi)\right)(\xi)=\left[S^{\prime}(1)-\delta_{1} / 2, S^{\prime}(1)+\delta_{1} / 2\right],
\end{gathered}
$$


and for $\xi \leq-X_{1}$,

$$
\begin{gathered}
\phi(\xi) \in\left[0, \delta_{1} / 4\right], \quad(h * S(\phi))(\xi) \in\left[S(0), S(0)+\delta_{1} / 2\right], \\
\left(h * S^{\prime}(\phi)\right)(\xi)=\left[S^{\prime}(0)-\delta_{1} / 2, S^{\prime}(0)+\delta_{1} / 2\right], \\
(h * \phi)(\xi) \max _{u \in[0,1]}\left|S^{\prime \prime}(u)\right| \in\left(0, \delta_{1} / 8\right) .
\end{gathered}
$$

Since $p(t)-c t-\omega \rightarrow 0$ as $t \rightarrow-\infty$, there exists $T_{5} \leq T$, where $T$ is defined in Lemma 4.1, such that for $t \leq T_{5}$,

$$
2[p(t)-c t-\omega] \max _{u \in[0,1]}\left|S^{\prime \prime}(u)\right| \cdot \max _{u \in[0,1]} \phi^{\prime}(u) \in\left(0, \delta_{1} / 8\right)
$$

Let $\kappa_{1}=\min _{\xi \in\left[-X_{1}, X_{1}\right]} \phi^{\prime}(\xi)>0$; then there exists a large $\sigma_{1}>0$ such that

$$
\frac{1}{2} c \sigma_{1} \varrho_{0} \kappa_{1}-\varrho_{0}-2 \max _{u \in[0,1], v \in[S(0), S(1)]}|g(u, v)| \geq 0 .
$$

Let $\Psi(x, t)=\phi(x+c t+\omega)+\phi(-x+c t+\omega)$. It is easy to prove that

$$
\lim _{t \rightarrow-\infty}\|\Phi-\Psi\|_{C^{0}(\mathbb{R} \times(-\infty, t])}=0 .
$$

By interpolation $\|\cdot\|_{C^{1}} \leq 2 \sqrt{\|\cdot\|_{C^{0}}\|\cdot\|_{C^{2}}}$; we have $\lim _{t \rightarrow-\infty}\|\Phi-\Psi\|_{C^{1}(\mathbb{R} \times(-\infty, t])}=$ 0 . Thus, there exists $T_{6} \leq T_{5}$ such that for any $t \leq T_{6}$,

$$
\|\Phi-\Psi\|_{C^{1}(\mathbb{R} \times(-\infty, t])} \leq \frac{1}{2} c \kappa_{1} .
$$

Since for each $t \in\left[T_{6}, T_{4}\right], \lim _{|x| \rightarrow+\infty} \Phi(x, t)=1, \lim _{|x| \rightarrow+\infty}(h * S(\Phi))(x, t)=$ $S^{\prime}(1)$ and $\lim _{|x| \rightarrow+\infty}\left(h * S^{\prime}(\Phi)\right)(x, t)=S^{\prime}(1)$, then there exists a large positive number $X_{2}$ such that for any $|x|>X_{2}$ and $t \in\left[T_{6}, T_{4}\right]$, (5.6) and (5.7) hold.

Let $\kappa_{2}=\min _{|x| \leq X_{2}, t \in\left[T_{6}, T_{4}\right]} \frac{\partial \Phi(x, t)}{\partial t}$. Take $\sigma_{2}>0$ such that

$$
\sigma_{2} \varrho_{0} \kappa_{2}-\varrho_{0}-2 \max _{u \in[0,1], v \in[S(0), S(1)]}|g(u, v)| \geq 0 .
$$

Now define

$$
\begin{aligned}
& A_{2}^{+}=\left\{(x, t): \Phi\left(x, r+t+\sigma \delta\left[1-e^{-\varrho_{0} t}\right]\right)+\delta e^{-\varrho_{0} t}>1,(x, t) \in \mathbb{R} \times[0,+\infty)\right\}, \\
& A_{2}^{-}=\left\{(x, t): \Phi\left(x, r+t+\sigma \delta\left[1-e^{-\varrho_{0} t}\right]\right)+\delta e^{-\varrho_{0} t}<1,(x, t) \in \mathbb{R} \times[0,+\infty)\right\} . \\
& \text { If }(x, t) \in A_{2}^{+} \text {, then } \\
& \mathcal{N}\left[W^{+}\right]:=\frac{\partial W^{+}}{\partial t}-d \Delta W^{+}-g\left(W^{+}(x, t),\left(h * S\left(W^{+}\right)\right)(x, t)\right) \geq-g(1, S(1))=0 .
\end{aligned}
$$


If $(x, t) \in A_{2}^{-}$, let $\xi(t)=r+t+\sigma \delta\left[1-e^{-\varrho_{0} t}\right]$; we have $W^{+}(x, t)=\Phi(x, \xi(t))+$ $\delta e^{-\varrho_{0} t}$. Then

$$
\begin{aligned}
& \mathcal{N}\left[W^{+}\right]=\delta e^{-\varrho_{0} t}\left[\varrho_{0} \sigma \Phi_{2}^{\prime}(x, \xi(t))-\varrho_{0}\right]-g\left(W^{+}(x, t),\left(h * S\left(W^{+}\right)\right)(x, t)\right) \\
&+g(\Phi(x, \xi(t)),(h * S(\Phi))(x, \xi(t))) \\
&= \delta e^{-\varrho_{0} t}\left[\varrho_{0} \sigma \Phi_{2}^{\prime}(x, \xi(t))-\varrho_{0}\right]-\int_{0}^{1}\left[\partial_{1} g\left(\Phi(x, \xi(t))+\theta \delta e^{-\varrho_{0} t}, \zeta(x, t)\right) \delta e^{-\varrho_{0} t}\right. \\
&+\partial_{2} g\left(\Phi(x, \xi(t))+\theta \delta e^{-\varrho_{0} t}, \zeta(x, t)\right) \\
&\left.\times \int_{0}^{\tau} \int_{-\infty}^{\infty} h(y, s) S^{\prime}(\Phi(x-y, \xi(t)-s)+\theta \eta(y, s)) \eta(y, s) d y d s\right] d \theta \\
& \geq \delta e^{-\varrho_{0} t}\left[\varrho_{0} \sigma \Phi_{2}^{\prime}(x, \xi(t))-\varrho_{0}\right]-\int_{0}^{1}\left[\partial_{1} g\left(\Phi(x, \xi(t))+\theta \delta e^{-\varrho_{0} t}, \zeta(x, t)\right) \delta e^{-\varrho_{0} t}\right. \\
&+\partial_{2} g\left(\Phi(x, \xi(t))+\theta \delta e^{-\varrho_{0} t}, \zeta(x, t)\right) \\
&\left.\times \int_{0}^{\tau} \int_{-\infty}^{\infty} h(y, s) S^{\prime}(\Phi(x-y, \xi(t)-s)+\theta \eta(y, s)) \delta e^{-\varrho_{0} t} e^{\varrho_{0} \tau} d y d s\right] d \theta \\
& \geq \delta e^{-\varrho_{0} t}\left\{\varrho_{0} \sigma \Phi_{2}^{\prime}(x, \xi(t))-\varrho_{0}-\int_{0}^{1}\left[\partial_{1} g\left(\Phi(x, \xi(t))+\theta \delta e^{-\varrho_{0} t}, \zeta(x, t)\right)\right.\right. \\
&+e^{\varrho_{0} \tau} \partial_{2} g\left(\Phi(x, \xi(t))+\theta \delta e^{-\varrho_{0} t}, \zeta(x, t)\right) \\
&\left.\left.\times\left(\left(h * S^{\prime}(\Phi)\right)(x, \xi(t))+\delta e^{\varrho_{0} \tau} \max _{u \in[0,1]}\left|S^{\prime \prime}(u)\right|\right)\right] d \theta\right\}
\end{aligned}
$$

where

$$
\begin{aligned}
\Phi_{2}^{\prime}(x, t) & =\frac{\partial}{\partial t} \Phi(x, t), \zeta(x, t) \\
& =\int_{0}^{\tau} \int_{-\infty}^{\infty} h(y, s) S(\Phi(x-y, \xi(t)-s)+\theta \eta(y, s)) d y d s
\end{aligned}
$$

and

$$
\begin{aligned}
\eta(y, s) & =W^{+}(x-y, t-s)-\Phi(x-y, \xi(t)-s) \\
& \leq \Phi(x-y, \xi(t-s))+\delta e^{-\varrho_{0}(t-s)}-\Phi(x-y, \xi(t)-s) \\
& \leq \Phi\left(x-y, \xi(t)-s+\sigma \delta e^{-\varrho_{0} t}\left[1-e^{\varrho_{0} s}\right]\right)+\delta e^{-\varrho_{0}(t-s)}-\Phi(x-y, \xi(t)-s) \\
& \leq \delta e^{-\varrho_{0}(t-s)} \leq \delta e^{-\varrho_{0} t} e^{\varrho_{0} \tau} \leq \delta e^{\varrho_{0} \tau} .
\end{aligned}
$$

Let $\sigma_{0}=\max \left\{\sigma_{1}, \sigma_{2}\right\}$. Now we consider six cases.

Case (i). $x \in \mathbb{R}, \xi(t)>T_{4}$. By (5.4), (5.5), (5.6) and (5.7), we have $\mathcal{N}\left[W^{+}\right] \geq 0$.

Case (ii). $\xi(t) \leq T_{6},|x|+c \xi(t)+\omega \geq X_{1}$. Since for $x>0$,

$\Phi(x, \xi(t)) \geq \phi(x+c \xi(t)+\omega), \quad \Phi(x-y, \xi(t)-s) \geq \phi(x-y+c \xi(t)-c s+\omega)$,

$\Phi(x-y, \xi(t)-s) \leq \phi(x-y+p(\xi(t)-s))+\phi(-x+y+p(\xi(t)-s))$,

then

$$
\begin{aligned}
& S^{\prime}(\Phi(x-y, \xi(t)-s)) \\
\leq & S^{\prime}(\phi(x-y+\xi(t)-s+\omega))+\phi(-x+y+c \xi(t)-c s+\omega) \max _{u \in[0,1]}\left|S^{\prime \prime}(u)\right| \\
& +2[p(\xi(t))-c \xi(t)-\omega] \max _{u \in[0,1]}\left|S^{\prime \prime}(u)\right| \cdot \max _{v \in \mathbb{R}} \phi^{\prime}(v) .
\end{aligned}
$$


Now by (5.4), (5.5), (5.8), (5.9), (5.12) and (5.13), for $\xi(t) \leq T_{6}, x>0$ with $x+c \xi(t)+\omega \geq X_{1}$, we have $\mathcal{N}\left[W^{+}\right] \geq 0$. By the symmetry, we have $\mathcal{N}\left[W^{+}\right] \geq 0$ for $\xi(t) \leq T_{6}$, and $x<0$ with $-x+c \xi(t)+\omega \geq X_{1}$.

Case (iii). $\xi(t) \leq T_{6},|x|+c \xi(t)+\omega \leq-X_{1}$. From (5.4), (5.5), (5.10), (5.11), (5.12) and (5.13), it follows that $\mathcal{N}\left[W^{+}\right] \geq 0$.

Case (iv). $\xi(t) \leq T_{6},-X_{1} \leq|x|+c \xi(t)+\omega \leq X_{1}$. By (5.14) and (5.15), we have $\mathcal{N}\left[W^{+}\right] \geq 0$.

Case (v). $T_{6} \leq \xi(t) \leq T_{4},|x|>X_{2}$. By (5.4), (5.5), (5.6) and (5.7), there is $\mathcal{N}\left[W^{+}\right] \geq 0$.

Case (vi). $T_{6} \leq \xi(t) \leq T_{4},|x| \leq X_{2}$. It is easy to see that (5.16) implies $\mathcal{N}\left[W^{+}\right] \geq 0$.

Combining the above six cases, we have proved that for $(x, t) \in A_{2}^{-}, \mathcal{N}\left[W^{+}\right] \geq 0$. Thus, as in Lemma 4.1, we can prove that $W^{+}(x, t)$ is a supersolution of (1.1) on $\mathbb{R} \times[0,+\infty)$.

Theorem 5.8. Assume that $h(x, t)=J(x) \delta(t-\tau)$; then for some $\left(x_{0}, t_{0}\right) \in \mathbb{R}^{2}$,

$$
\widetilde{\Phi}(x, t)=\Phi\left(x+x_{0}, t+t_{0}\right) \text { for any }(x, t) \in \mathbb{R}^{2} .
$$

Proof. Fix an arbitrary $t_{0} \in \mathbb{R}$. Define

$$
\eta:=\inf _{z \in \mathbb{R}, r \in \mathbb{R}}\left\|\Phi(\cdot, r+\cdot)-\widetilde{\Phi}\left(\cdot+z, t_{0}+\cdot\right)\right\|_{L^{\infty}(\mathbb{R} \times[-\tau, 0])} .
$$

Fix any small $\delta \in\left(0, \delta_{0}\right]$. By Lemma [5.6, there exist $t_{1}<t_{0}, z \in \mathbb{R}$ and $r \in \mathbb{R}$ such that

$$
\left\|\Phi(\cdot, r+\cdot)-\widetilde{\Phi}\left(\cdot+z, t_{1}+\cdot\right)\right\|_{L^{\infty}(\mathbb{R} \times[-\tau, 0])} \leq \delta .
$$

That is, $\Phi(x, r+s)-\delta \leq u\left(z+x, t_{1}+s\right) \leq \Phi(x, r+s)+\delta$ for any $x \in \mathbb{R}$ and $s \in[-\tau, 0]$. Furthermore,

$$
\begin{aligned}
& \max \left\{\Phi\left(x, r+\sigma \delta\left[1-e^{\varrho_{0} \tau}\right]+s-\sigma \delta\left[1-e^{-\varrho_{0} s}\right]\right)-\delta e^{-\varrho_{0} s}, 0\right\} \\
& \leq \widetilde{\Phi}\left(z+x, t_{1}+s\right) \leq \min \left\{\Phi \left(x, r-\sigma \delta\left[1-e^{\varrho_{0} \tau}\right]\right.\right. \\
& \left.\left.\quad+s+\sigma \delta\left[1-e^{-\varrho_{0} s}\right]\right)+\delta e^{-\varrho_{0} s}, 1\right\} .
\end{aligned}
$$

By comparison, for all $t \geq 0$,

$$
\begin{aligned}
& \max \left\{\Phi\left(x, r+\sigma \delta\left[1-e^{\varrho_{0} \tau}\right]+t-\sigma \delta\left[1-e^{-\varrho_{0} t}\right]\right)-\delta e^{-\varrho_{0} t}, 0\right\} \\
& \leq \widetilde{\Phi}\left(z+x, t_{1}+t\right) \leq \min \left\{\Phi \left(x, r-\sigma \delta\left[1-e^{\varrho_{0} \tau}\right]\right.\right. \\
& \left.\left.+t+\sigma \delta\left[1-e^{-\varrho_{0} t}\right]\right)+\delta e^{-\varrho_{0} t}, 1\right\} .
\end{aligned}
$$

Set $t=t_{0}-t_{1}$ and $r^{\prime}=r+\sigma \delta\left[1-e^{\varrho_{0} \tau}\right]-\sigma \delta\left[1-e^{-\varrho_{0} t}\right]$. We then have

$$
\begin{aligned}
\left|\widetilde{\Phi}\left(z+x, t_{0}\right)-\Phi\left(x, r^{\prime}\right)\right| & \leq 2 \delta+\left|\Phi\left(x, r^{\prime}+\left(1+e^{\varrho_{0} \tau}\right) \sigma \delta\right)-\Phi\left(x, r^{\prime}\right)\right| \\
& \leq\left(2+\left(1+e^{\varrho_{0} \tau}\right) \sigma\left\|\frac{\partial \Phi}{\partial t}\right\|_{\infty}\right) \delta .
\end{aligned}
$$

Thus, $\eta \leq\left(2+\left(1+e^{\varrho_{0} \tau}\right) \sigma\left\|\frac{\partial \Phi}{\partial t}\right\|_{\infty}\right) \delta$. Since $\delta$ is arbitrary, $\eta=0$. Consequently, $\widetilde{\Phi}$ is a translation of $\Phi$. This completes the proof.

Remark 5.9. We note that the assumption $h(x, t)=J(x) \delta(t-\tau)$ is only used to ensure that (5.2) holds. Obviously, if $h(x, t)$ satisfies that there exists $\tau_{0} \in(0, \tau)$ such that $\int_{\tau_{0}}^{\tau} \int_{-\infty}^{\infty} h(x, t) d x d t=1$, that is, $\int_{0}^{\tau_{0}} \int_{-\infty}^{\infty} h(x, t) d x d t=0$, we can show that (5.2) holds via a similar argument to that of Lemma 5.5. Thus, the conclusions 
of Lemma 5.6 and Theorem 5.8 are still valid if $h(x, t)$ satisfies that there exists $\tau_{0} \in(0, \tau)$ such that $\int_{\tau_{0}}^{\tau} \int_{-\infty}^{\infty} h(x, t) d x d t=1$. However, it seems difficult to show that (5.2) holds for a general kernel $h(x, t)$. We also note that the assertions of Lemmas 5.15 .4 and 5.7 hold for a general kernel $h(x, t)$ satisfying (H1)-(H3).

Theorem 5.10. The entire solution $\Phi$ of (1.1) founded in Theorem 4.4 is Liapunov stable.

Proof. Given any $\epsilon>0$, for any $\varphi \in C_{[0,1]}$ with $\left\|\varphi-\Phi\left(x_{0}+\cdot, t_{0}+\cdot\right)\right\|_{L^{\infty}(\mathbb{R} \times[-\tau, 0])}$ $<\delta \leq \delta_{0}$, where $x_{0} \in \mathbb{R}$ and $t_{0} \in \mathbb{R}$ are arbitrary constants, we have

$$
\begin{aligned}
& \max \left\{\Phi\left(x+x_{0}, s+t_{0}+\sigma_{0} \delta\left(1-e^{\varrho_{0} \tau}\right)-\sigma_{0} \delta\left(1-e^{-\varrho_{0} s}\right)\right)-\delta e^{-\varrho_{0} s}, 0\right\} \\
\leq & \varphi(x, s) \leq \min \left\{\Phi\left(x+x_{0}, s+t_{0}-\sigma_{0} \delta\left(1-e^{\varrho_{0} \tau}\right)+\sigma_{0} \delta\left(1-e^{-\varrho_{0} s}\right)\right)+\delta e^{-\varrho_{0} s}, 1\right\}
\end{aligned}
$$

for all $x \in \mathbb{R}$ and $s \in[-\tau, 0]$, where $\varrho_{0}, \sigma_{0}$ and $\delta_{0}$ are as in Lemma 5.7. By Lemma 5.7, it follows that

$$
\begin{aligned}
\max \left\{\Phi \left(x+x_{0}, t+t_{0}\right.\right. & \left.\left.+\sigma_{0} \delta\left(1-e^{\varrho_{0} \tau}\right)-\sigma_{0} \delta\left(1-e^{-\varrho_{0} t}\right)\right)-\delta e^{-\varrho_{0} t}, 0\right\} \\
\leq \quad u(x, t ; \varphi) \leq \min \{\Phi(x & +x_{0}, t+t_{0}-\sigma_{0} \delta\left(1-e^{\varrho_{0} \tau}\right) \\
& \left.\left.+\sigma_{0} \delta\left(1-e^{-\varrho_{0} t}\right)\right)+\delta e^{-\varrho_{0} t}, 1\right\}
\end{aligned}
$$

for all $x \in \mathbb{R}$ and $t>0$. Choose $\delta_{2}(\epsilon)>0$ such that $\|\Phi(\cdot, t)-\Phi(\cdot, t+z)\|_{L^{\infty}(\mathbb{R})}<$ $\epsilon / 2$ for any $|z| \leq \delta_{2}$ and $t \in \mathbb{R}$. Furthermore, let $\delta^{*}=\min \left\{\epsilon / 2, \frac{\delta_{2} e^{-\varrho_{0} \tau}}{\sigma_{0}}, \delta_{0}\right\}$. Then for any $\delta<\delta^{*}$,

$$
\left|\sigma_{0} \delta\left(1-e^{\varrho_{0} \tau}\right)-\sigma_{0} \delta\left(1-e^{-\varrho_{0} t}\right)\right| \leq\left|\sigma_{0} \delta\left(e^{\varrho_{0} \tau}-e^{-\varrho_{0} t}\right)\right| \leq \sigma_{0} \delta e^{\varrho_{0} \tau} \leq \delta_{2} .
$$

It follows that

$$
\Phi\left(x+x_{0}, t+t_{0}\right)-\epsilon \leq u(x, t ; \varphi) \leq \Phi\left(x+x_{0}, t+t_{0}\right)+\epsilon \quad \forall x \in \mathbb{R}, t \geq 0 .
$$

That is, for any $\varphi \in C_{[0,1]}$ with $\left\|\varphi-\Phi\left(\cdot+x_{0}, \cdot+t_{0}\right)\right\|_{L^{\infty}(\mathbb{R} \times[-\tau, 0])}<\delta^{*}$, we have

$$
\left|u(x, t ; \varphi)-\Phi\left(x+x_{0}, t+t_{0}\right)\right| \leq \epsilon
$$

for all $x \in \mathbb{R}$ and $t \geq 0$, which implies that $\Phi(x, t)$ is Liapunov stable.

Remark 5.11. To prove Theorem 1.1, we only need to let

$$
\hat{\Phi}(x, t)=\Phi\left(x+\left(\theta_{1}-\theta_{2}\right) / 2, t+\left(\theta_{1}+\theta_{2}-2 \omega\right) /(2 c)\right)
$$

and still denote $\hat{\Phi}(x, t)$ by $\Phi(x, t)$.

Remark 5.12. Consider the case $c<0$. Assume that $\phi(x+c t)$ is an increasing traveling wave solution up to translation of (1.1) satisfying $\phi(-\infty)=0$ and $\phi(+\infty)=1$. Let $\psi(x-c t)=\phi(-(x-c t))$. Then $\psi(-\infty)=1$ and $\psi(+\infty)=0$. Let $c^{\prime}=-c>0$ and $\chi\left(x+c^{\prime} t\right)=1-\psi\left(x+c^{\prime} t\right)=1-\psi(x-c t)$. Thus, $\chi(-\infty)=0$ and $\chi(\infty)=1$. We conclude that $\chi\left(x+c^{\prime} t\right)$ is a traveling wave solution of the following equation:

$$
\frac{\partial u}{\partial t}=d \Delta u-g(1-u(x, t),(h * S(1-u))(x, t)) .
$$

Take $g^{*}(u, v)=-g(1-u,-v)$ and $S^{*}(u)=-S(1-u)$. Obviously, $g^{*}$ and $S^{*}$ satisfy the conditions (F1) and (F2). Then equation (5.17) reduces to

$$
\frac{\partial u}{\partial t}=d \Delta u+g^{*}\left(u,\left(h * S^{*}(u)\right)(x, t)\right) .
$$

Applying Theorem 1.1 to (5.18), we can prove Theorem 1.2 


\section{REFERENCES}

[1] S. Ai, Travelling wave fronts for generalized Fisher equations with spatio-temporal delays, J. Differential Equations 232 (2007), 104-133. MR2281191 (2007h:34052)

[2] P. B. Ashwin, M. V. Bartuccelli, T. J. Bridges and S. A. Gourley, Travelling fronts for the KPP equation with spatio-temporal delay, Z. Angew. Math. Phys. 53 (2002), 103-122. MR.1889183 (2002m:35097)

[3] J. F. M. Al-Omari and S. A. Gourley, A nonlocal reaction-diffusion model for a single species with stage structure and distributed maturation delay, Euro. J. Appl. Math. 16 (2005), 37-51. MR2148679 (2006c:35146)

[4] J. Billingham, Dynamics of a strongly nonlocal reaction-diffusion population model, Nonlinearity 17 (2004), 313-346. MR2023445 (2004m:35142)

[5] N.F. Britton, Spatial structures and periodic travelling waves in an integro-deferential reaction-diffusion population model, SIAM J. Appl. Math. 50 (1990), 1663-1688. MR.1080515 (91m:92020)

[6] J. Carr and A. Chmaj, Uniqueness of travelling waves for nonlocal monostable equations, Proc. Amer. Math. Soc. 132 (2004), 2433-2439. MR2052422 (2005b:35125)

[7] X. Chen, Existence, uniqueness, and asymptotic stability of travelling waves in nonlocal evolution equations, Adv. Differential Equations 2 (1997), 125-160. MR1424765 (98f:35069)

[8] X. Chen and J. S. Guo, Existence and uniqueness of entire solutions for a reaction-diffusion equation, J. Differential Equations 212 (2005), 62-84. MR2130547 (2006b:35179)

[9] X. Chen, J. S. Guo and H. Ninomiya, Entire solutions of reaction-diffusion equations with balanced bistable nonlinearities, Proc. Roy. Soc. Edinburgh Sect. A 136 (2006), 1207-1237. MR 2290131 (2007k:35256)

[10] D. Daners and P. K. McLeod, Abstract evolution equations, periodic problems and applications, Pitman Res. Notes Math. Ser. 279, Longman Scientific and Technical, Harlow, 1992.

[11] O. Diekmann and H. G. Kaper, On the bounded solutions of a nonlinear convolution equation, Nonlinear Analysis TMA 2 (1978), 721-737. MR512163 (80c:45015)

[12] P. C. Fife and J. B. McLeod, The approach of solutions of nonlinear diffusion equations to traveling wave solutions, Arch. Rational Mech. Anal. 65 (1977), 335-361. MR0442480 $(56: 862)$

[13] P. C. Fife and J. B. McLeod, A phase plane discussion of convergence to travelling fronts for nonlinear diffusion, Arch. Rational Mech. Anal. 75 (1980/81), 281-314. MR607901 (83b:35085)

[14] T. Faria, W. Huang and J. Wu, Traveling waves for delayed reaction-diffusion equations with global response, Proc. R. Soc. Lond. 462A (2006), 229-261. MR2189262 (2006i:35182)

[15] T. Faria and S. Trofimchuk, Nonmonotone travelling waves in a single species reactiondiffusion equation with delay, J. Differential Equations 228 (2006), 357-376. MR2254435 (2007f:35159)

[16] Y. Fukao, Y. Morita and H. Ninomiya, Some entire solutions of the Allen-Cahn equation, Taiwanese J. Math. 8 (2004), 15-32. MR2057634 (2005a:35159)

[17] S. A. Gourley and Y. Kuang, Wavefronts and global stability in a time-delayed population model with stage structure, Proc. Roy. Soc. Lond. 459A (2003), 1563-1579. MR 1994271 (2004f:92024)

[18] S. A. Gourley and S. Ruan, Convergence and travelling fronts in functional differential equations with nonlocal terms: a competition model, SIAM J. Math. Anal. 35 (2003), 806-822. MR2048407 (2005i:35142)

[19] S. A. Gourley, J. H. W. So and J. Wu, Non-locality of reaction-diffusion equations induced by delay: biological modeling and nonlinear dynamics, J. Math. Sci. 124 (2004), 5119-5153. MR2129130 (2006b:35182)

[20] J. S. Guo and Y. Morita, Entire solutions of reaction-diffusion equations and an application to discrete diffusive equations, Discrete Cont. Dyn. Systems 12 (2005), 193-212. MR2122162 (2006f:35145)

[21] F. Hamel and N. Nadirashvili, Entire solutions of the KPP Equation, Comm. Pure Appl. Math. LII (1999), 1255-1276. MR1699968 (2000e:35088)

[22] F. Hamel and N. Nadirashvili, Travelling fronts and entire solutions of the Fisher-KPP equation in $\mathbb{R}^{N}$, Arch. Rational Mech. Anal. 157 (2001), 91-163. MR1830037(2002d:35090) 
[23] W. T. Li, G. Lin and S. Ruan, Existence of traveling wave solutions in delayed reactiondiffusion systems with applications to diffusion-competition systems, Nonlinearity 19 (2006), 1253-1273. MR 2229998 (2007a:92055)

[24] W. T. Li and Z. C. Wang, Traveling fronts in diffusive and cooperative Lotka-Volterra system with nonlocal delays, Z. angew. Math. Phys. 58 (2007), 571-591. MR2341686

[25] D. Liang and J. Wu, Travelling waves and numerical approximations in a reaction advection diffusion equation with nonlocal delayed effects, J. Nonlinear Sci. 13 (2003), 289-310. MR.1982017 (2004d:92024)

[26] S. Ma and J. Wu, Existence, uniqueness and asymptotic stability of traveling wavefronts in non-local delayed diffusion equation, J. Dynam. Differential Equations 19 (2007), 391-436. MR2333414

[27] R. H. Martin and H. L. Smith, Abstract functional differential equations and reactiondiffusion systems, Trans. Amer. Math. Soc. 321 (1990), 1-44. MR.967316 (90m:35194)

[28] Y. Morita and H. Ninomiya, Entire solutions with merging fronts to reaction-diffusion equations, J. Dynam. Differential Equations 18 (2006), 841-861. MR2263404 (2007h:35192)

[29] C. Ou and J. Wu, Persistence of wavefronts in delayed non-local reaction diffusion equations, J. Differential Equations 235 (2007), 216-239. MR2309573 (2008c:35151)

[30] A. Pazy, Semigroups of linear operators and applications to partial differential equations, Springer-Verlag, New York, 1983. MR710486 (85g:47061)

[31] P. Poláčik, Convergence in smooth strongly monotone flows defined by semilinear parabolic equations, J. Differential Equations 79 (1989), 89-110. MR997611 (90f:58025)

[32] P. Poláčik, Domains of attraction of equilibria and monotonicity properties of convergent trajectories in parabolic systems admitting strong comparison principle, J. Reine Angew. Math. 400 (1989), 32-56. MR1013724 (90i:58099)

[33] M. H. Protter and H. F. Weinberger, Maximum principles in differential equations, PrenticeHall, 1983. MR0219861 (36:2935)

[34] S. Ruan, Spatial-temporal dynamics in nonlocal epidemiological models, in "Mathematics for Life Science and Medicine", Y. Iwasa, K. Sato and Y. Takeuchi, eds., Springer-Verlag, New York, 2007, pp. 99-122. MR2309365

[35] S. Ruan and D. Xiao, Stability of steady states and existence of traveling waves in a vector disease model, Proc. Roy. Soc. Edinburgh Sect. A 134 (2004), 991-1011. MR2099575 (2005i:35152)

[36] K. W. Schaaf, Asymptotic behavior and travelling wave solutions for parabolic functional differential equations, Trans. Amer. Math. Soc. 302 (1987), 587-615. MR891637 (88g:35190)

[37] H. L. Smith, Monotone dynamical systems: an introduction to the theory of competitive and cooperative systems, Mathematical Surveys and Monographs, 41, Amer. Math. Soc., Providence, RI, 1995. MR1319817 (96c:34002)

[38] H. L. Smith and X. Q. Zhao, Global asymptotic stability of travelling waves in delayed reaction-diffusion equations, SIAM J. Math. Anal. 31 (2000), 514-534. MR 1740724 (2001c:35239)

[39] A. I. Volpert, V. A. Volpert and V. A. Volpert, Travelling wave solutions of parabolic systems, Translations of Mathematical Monographs, 140, Amer. Math. Soc., Providence, RI, 1994. MR:1297766 (96c:35092)

[40] Z. C. Wang and W. T. Li, Monotone travelling fronts of a food-limited population model with nonlocal delay, Nonlinear Analysis RWA 8(2007), 699-712. MR2289581 (2007i:35129)

[41] Z. C. Wang, W. T. Li and S. Ruan, Travelling wave fronts of reaction-diffusion systems with spatio-temporal delays, J. Differential Equations 222 (2006), 185-232. MR2200751 (2006k:35156)

[42] Z. C. Wang, W. T. Li and S. Ruan, Existence and stability of traveling wave fronts in reaction advection diffusion equations with nonlocal delay, J. Differential Equations 238 (2007), 153200. MR2334595

[43] Z. C. Wang, W. T. Li and S. Ruan, Traveling fronts in monostable equations with nonlocal delayed effects, J. Dynamics and Differential Equations 20 (2008), 563-607.

[44] D. V. Widder, The Laplace transform, Princeton University Press, Princeton, NJ, 1941. MR0005923 (3:232d)

[45] J. Wu, Theory and applications of partial functional differential equations, Springer-Verlag, New York, 1996. MR1415838(98a:35135) 
[46] J. Wu and X. Zou, Travelling wave fronts of reaction-diffusion systems with delay, J. Dynam. Differential Equations 13 (2001), 651-687. (Erratum, J. Dynam. Differential Equations, DOI: 10.1007/s10884-007-9090-1.) MR1845097 (2003a:35114)

[47] H. Yagisita, Backward global solutions characterizing annihilation dynamics of travelling fronts, Publ. Res. Inst. Math. Sci. 39 (2003), 117-164. MR 1935462 (2003h:35142)

[48] X. Zou, Delay induced traveling wave fronts in reaction diffusion equations of KPP-Fisher type, J. Comp. Appl. Math. 146 (2002), 309-321. MR1925963(2004c:35233)

School of Mathematics and Statistics, Lanzhou University, Lanzhou, Gansu 730000 , People's Republic of China

E-mail address: wangzhch@lzu.edu.cn

School of Mathematics and Statistics, Lanzhou University, Lanzhou, Gansu 730000 , People's Republic of China

E-mail address: wtli@lzu.edu.cn

Department of Mathematics, University of Miami, P.O. Box 249085, Coral Gables, FLORIDA 33124-4250

E-mail address: ruan@math.miami.edu 